\title{
Large-scale Evolutionary Multi-objective Optimization Assisted by Directed Sampling
}

\author{
Shufen Qin, Chaoli Sun, Member, IEEE, Yaochu Jin, Fellow, IEEE, Ying Tan, \\ and Jonathan Fieldsend, Member, IEEE
}

\begin{abstract}
It is particularly challenging for evolutionary algorithms to quickly converge to the Pareto front in large-scale multi-objective optimization. To tackle this problem, this paper proposes a large-scale multi-objective evolutionary algorithm assisted by some selected individuals generated by directed sampling. At each generation, a set of individuals closer to the ideal point are chosen for performing a directed sampling in the decision space, and those non-dominated ones of the sampled solutions are used to assist the reproduction to improve the convergence in evolutionary large-scale multi-objective optimization. In addition, elitist non-dominated sorting is adopted complementarily for environmental selection with a reference vector based method in order to maintain diversity of the population. Our experimental results show that the proposed algorithm is highly competitive on large-scale multi-objective optimization test problems with up to 5000 decision variables compared to five state-of-the-art multi-objective evolutionary algorithms.
\end{abstract}

Index Terms-Evolutionary multi-objective optimization, large-scale multi-objective problems, directed sampling, nondominated sorting, reference vectors.

\section{INTRODUCTION}

A Large number of multi-objective evolutionary algorithms (MOEAs) [1]-[8] have been proposed in the past decades for solving multi-objective optimization problems (MOPs). They can roughly be divided into four classes [9], [10]: Pareto dominance based MOEAs [11]-[22], decomposition based MOEAs [23]-[28], performance indicator based MOEAs [29][38], and finally those MOEAs that do not fall in the previous three categories [39]-[42]. In recent years, most research on MOEAs [7], [17]-[21], [28], [36], [39], [40], [43]-[47] has focused on the scalability of the performance to the increase in the number of objectives, and little attention has been paid to the scalability of the performance in the decision space. However, many real-world multi-objective optimization problems may have hundreds or even thousands of decision

Shufen Qin is with the School of Electronic Information Engineering, Taiyuan University of Science and Technology, Taiyuan 030024, China. email: shufen.qin@stu.tyust.edu.cn.

Chaoli Sun is with the Department of Computer Science and Technology, Taiyuan University of Science and Technology, Taiyuan 030024, China. email: chaoli.sun@tyust.edu.cn. (Corresponding author).

Yaochu Jin is with the Department of Computer Science, University of Surrey, Guildford, GU2 7XH, UK. e-mail: yaochu.jin@ surrey.ac.uk. (Corresponding author).

Ying Tan is with the Department of Computer Science and Technology, Taiyuan University of Science and Technology, Taiyuan 030024, China. email: tanying2015@163.com.

Jonathan Fieldsend is with the Department of Computer Science, University of Exeter, Devon, EX4 4QF, UK. e-mail: J.E.Fieldsend@exeter.ac.uk. variables, which are known as large-scale optimization problems [48]. For example, a flight safety system has over 1500 parameters [49], and in the search of optimal structure of deep neural networks, the number of weight and structure parameters dramatically increase as the number of hidden nodes and the number of hidden layers increase [50], [51].

The performance of classic metaheuristic algorithms often deteriorates as the dimension of the decision space increases [52], which is called curse of dimensionality [53]. Although large-scale single-objective optimization has been a popular research topic for many years [54]-[58], research on large-scale multi-objective optimization is still in its early stage [52], [59]-[68]. Generally, existing approaches to largescale multi-objective problems (LSMOPs) can be roughly classified into four categories:

1) Cooperative coevolution (CC) framework based MOEAs. Antonio and Coello Coello applied a differential evolution algorithm (GDE3) in the cooperative coevolution framework for solving large-scale many-objective problems [59], and then they further proposed to combine MOEA/D with coevolutionary techniques for decomposition in both objective and decision spaces [61]. Li et al. [64] also proposed a cooperative coevolutionary algorithm, in which a fast interdependency identification grouping method was utilized, for large-scale multi-objective problems.

2) Decision variable clustering based MOEAs. Ma et al. [62] divided the decision variables into three groups according to their properties related to convergence and diversity, and proposed a multi-objective evolutionary algorithm based on decision variable analyses (MOEA/DVA). Zhang et al. [52] extended the idea of MOEA/DVA [62] and proposed an evolutionary algorithm for large-scale many-objective optimization (LMEA) by dividing the decision variables more precisely and introducing different search and selection strategies for different groups. Liu et al. [69] proposed to group large-scale decision variables into two categories, i.e., convergence-related and diversity-related variables. Then principal components analysis (PCA) is performed to reduce the dimension of the convergence-related variables, which will further be decomposed into a number of sub-problems based on interdependence analysis for optimization. Chen et al. also separated the variables into convergence-related and diversity-related clusters, 
and proposed to optimize sub-problems in parallel [63] and sequentially [70], respectively. Note, however, that such algorithms often require a large number of fitness evaluations to achieve adequately precise clustering of the decision variables.

3) Problem transformation based MOEAs. Zille et al. [65] proposed a framework for large-scale multi-objective optimization (WOF) using a problem transformation technique to reduce the search space, which can serve as a genetic method for any population-based metaheuristic algorithms. Following that, He et al. [66] implemented a dimension reduction method by optimizing a set of weight variables along different directions in the decision space. Most recently, He et al. [71] further proposed an adaptive offspring generation method for large-scale optimization problems. Liu et al. [72] proposed to use a random dynamic grouping instead of ordered grouping to improve the performance of the WOF framework [65].

4) New learning strategies based MOEAs. Not so many methods falling in this category have been proposed. Tian et al. [67] proposed a modified competitive swarm optimizer for large-scale multi-objective problems, called LMOCSO, by generating promising offspring to accelerate the search of the global optimum. Zhang et al. [73] utilized the information of the previous positions of the population, and built an information feedback model (IFM) to generate an offspring population.

Although existing large-scale optimization algorithms have showed promising performances, each category of the algorithms has its own drawback. For example, some cooperative coevolution and clustering techniques based MOEAs need to classify the decision variables into a set of groups, which will cost a great number of objective evaluations in order to detect interactive decision variables. Furthermore, the performance of the CC framework based MOEAs will seriously deteriorate on inappropriate groupings. In addition, the assumption of separability between groups of decision variables does not always hold. Therefore, the grouping strategies, such as linear grouping, random grouping, or ordered grouping, which do not cost additional objective evaluations to detect interactive decision variables, are not applicable for solving large-scale multiobjective optimization problems with interactions between all decision variables. The new learning strategy based MOEAs also need to cost a large number of evaluations to obtain better solutions, for example, $15000 \times D$ evaluations are required in LMOCSO [67] to obtain better solutions. The problem transformation based MOEAs are very competitive in enhancing the convergence capability for large-scale optimization, however, they are susceptible to local optima, as pointed out in [65], [66] and [67]. Moreover, the grouping scheme severely restricts the flexibility of the method given in [65].

Based on the above discussions, we can see that it is vital for an MOEA to be able to search in the right direction to achieve a set of good solutions with a limited computational budget for large-scale optimization problems. For this purpose, this work proposes a large-scale evolutionary multi-objective algorithm assisted by directed sampling, termed LMOEA-DS for short. The main idea is to estimate the promising search directions in the decision space, which is similar to the idea presented in [66]. Different to the method in [66] that uses non-dominated solutions of the current parent population to generate search directions in the decision space, in this work, by contrast, we use solutions closest to the ideal point in subpopulations divided based on a set of reference vectors. Furthermore, promising solutions are generated by random sampling along the estimated search directions, instead of performing an optimization as done in [66]. The non-dominated solutions of the sampled solutions are then used for reproduction. This above process of identifying promising solutions using directed sampling is repeated in each generation. In [66], by contrast, the promising solutions are used to seed the initial population and then an arbitrary existing MOEA can be used to search for a set of non-dominated solutions. The main contributions of this work are summarized as follows.

1) At each generation of the evolutionary search, a directed sampling strategy is adopted for generating promising solutions to guide the search. For this purpose, a set of reference vectors are adopted to group the population into a number of clusters. Then the individual having the minimum distance to the ideal point in each cluster will be used to generate two promising search directions in the decision space. A certain number of solutions are then randomly sampled along the two search directions. After that, the sampled solutions are combined and the non-dominated solutions among them are chosen for reproduction. These non-dominated solutions are called guiding solutions.

2) To strike a balance between convergence and diversity, two reproduction processes, each of which is followed by the proposed environmental selection, are introduced. In the first reproduction process, the guiding solutions are used to perform crossover with the current parent individuals, followed by the second reproduction, in which no guiding solutions will take part in the generation of offspring individuals.

3) Reference vectors and elitist non-dominated sorting are complementarily used in the environmental selection. By default, a set of reference vectors is used to decompose the MOP and both the Euclidean distance and anglebased metric are employed for selection for each subproblem. However, in case the population diversity is lost, i.e., when a large number of reference vectors are empty, the elitist non-dominated sorting strategy [12] will replace the reference based selection, aiming to promote the diversity of the population.

Section II describes the main framework and details of the proposed LMOEA-DS algorithm. In Section III, the test problems used in our experiments, the performance indicator for measuring the performance of the obtained non-dominated solution sets are presented, followed by a description of the experimental settings and the comparative results. Finally, conclusions are drawn and future work is outlined in Section IV. 


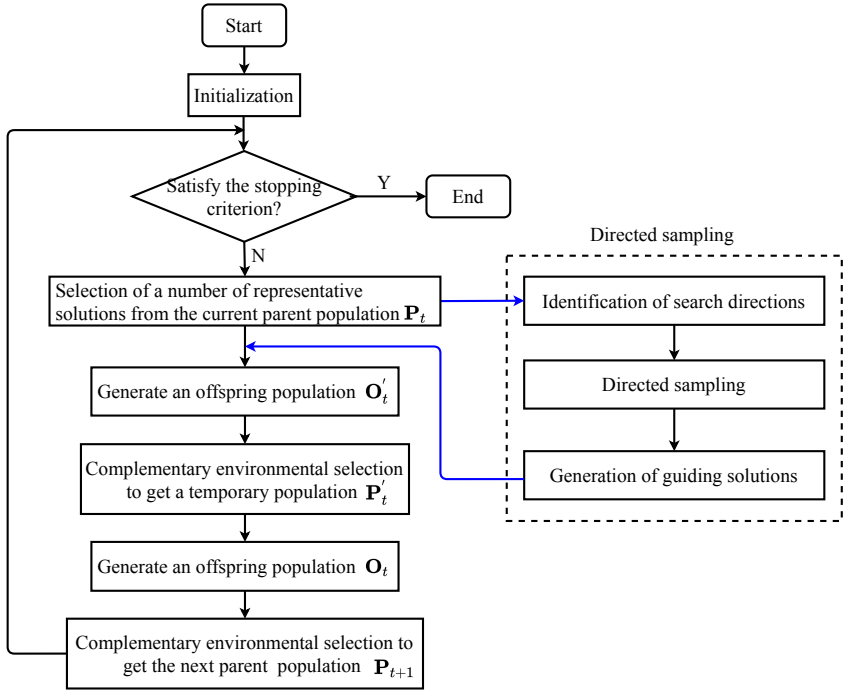

Fig. 1. A diagram of the overall framework of the proposed LMOEA-DS, which consists of three main components, namely generation of the guiding solutions, double reproduction, and complementary environmental selection.

\section{THE PROPOSED ALGORITHM}

Without loss of generality, a multi-objective minimization problem can be formulated as follows:

$$
\begin{aligned}
\min & \mathbf{F}(\mathbf{x})=\left(f_{1}(\mathbf{x}), f_{2}(\mathbf{x}), \ldots, f_{m}(\mathbf{x})\right) \\
\text { s.t. } & \mathbf{x} \in \mathbb{R}^{D}
\end{aligned}
$$

where $m$ is the number of objectives and $\mathbf{x}=\left(x_{1}, x_{2}, \ldots, x_{D}\right)$ is the decision vector, where $D$ is the dimension of the decision space. In this work, we consider bi- and threeobjective problems with a large number of decision variables, which may range from hundreds to thousands [66].

The search space exponentially increases when the number of decision variables increases, dramatically worsening the search performance, in particular the convergence capability of optimization algorithms. In order to address this issue, we propose a directed sampling strategy in combination with a double-reproduction strategy to accelerate the convergence, together with a new environmental selection method to promote population diversity. Fig. 1 depicts the flowchart of the overall framework of the proposed LMOEA-DS, which is composed of three main components: identification of the guiding solutions, guided double reproduction, and complementary environmental selection. Note that two complementary environmental selection strategies are provided to accelerate convergence while maintaining the diversity of the population. From Fig. 1, we can see that guiding solutions are embedded in the reproduction process in each generation, which is substantially different from [66], where the promising solutions found are used only for population initialization. In addition, the method for identifying the promising search directions is also different. In the following, we will detail the three main components of LMOEA-DS.

\section{A. Initialization}

The population is randomly initialized, like in most MOEAs. In addition, two sets of reference vectors $\mathbf{W}$ and $\mathbf{W}^{\prime}$ are created, which partition the objective space into sub-spaces for environmental selection and for generation of the search directions, respectively. Note that the number of reference vectors for generating the search directions should usually be considerably smaller than that for environmental selection to reduce additional computational costs introduced by the directed sampling. Suppose that $N_{w^{\prime}}$ solutions will be selected for generating the search directions, we simply group W reference vectors for environmental selection into $N_{w^{\prime}}-m$ clusters using k-means [74] and then add the $m$ boundary reference vectors in $\mathbf{W}$.

\section{B. Directed Sampling and Generation of Guiding Solutions}

Guiding solutions are meant to assist the MOEA to find the right search direction to accelerate the convergence. Here, we hypothesize that solutions that are closest to the ideal point in the current population are able to provide information about the desired search directions. However, it may be harmful to the diversity if only the closest solution for the whole population is used to guide the search. Therefore, we divide the overall search space into a number of subspaces using the reference vector set $\mathbf{W}^{\prime}$. The method for determining the search directions and guiding solutions will be elaborated below.

1) Identification of Search Directions: Recall that the search is performed in the decision space and consequently, we aim to identify promising search directions in the decision space. To this end, we identify the solutions closest to the ideal point of the objective space at first and then define the promising search directions by setting out a line from the lower or upper bound points to these solutions in the decision space. Note that the objective of using two directions is to increase the chance for the direction vectors to intersect with the Pareto set in the decision space. The experimental results of the methods that only use the direction setting out from $\mathbf{L}$ and that only use the direction setting out from $\mathbf{U}$, respectively, are given and analyzed in Section A of the Supplementary material. As previously mentioned, we firstly group the current parent population into $N_{w^{\prime}}$ subpopulations by assigning each solution to its closest reference vector of $\mathbf{W}^{\prime}$. Then, for each reference vector $\mathbf{w}_{i}^{\prime}$, where $i=1,2, \ldots, N_{w^{\prime}}$, to which at least one solution is assigned, the solution that has the minimum projected distance to the ideal point is chosen to define two search directions. To illustrate how the search directions are defined, an example is given in Fig. 2(a), where the current population has six solutions denoted by black dots, $\mathbf{x}_{1}, \mathbf{x}_{2}, \ldots, \mathbf{x}_{6}$ and $\mathbf{W}^{\prime}$ has four reference vectors. The blue dotted ellipse in Fig. 2(a) shows that the solutions in each ellipse are assigned to the same reference vector. The green dotted line indicates the distance of a solution to the origin along the related reference vector in the objective space. Note that reference vector $\mathbf{w}_{3}^{\prime}$ has no solutions assigned to it in this example. To address this issue, the solution, which is closest to the reference vector $\mathbf{w}_{3}^{\prime}$ among all individuals that have not been chosen to define the search directions, will be assigned to $\mathbf{w}_{3}^{\prime}$ so that each reference vector has at least one solution assigned to. For example, in Fig. 2(a), vector $\mathbf{w}_{3}^{\prime}$ has 
no solutions assigned to and $\mathbf{x}_{4}$ is the closest solution to it among $\mathbf{x}_{3}, \mathbf{x}_{4}$ and $\mathbf{x}_{5}$, which are not the closest solutions in their own clusters. In this case, solution $\mathbf{x}_{4}$ will be chosen to define the search directions for vector $\mathbf{w}_{3}^{\prime}$.

As a result, $2 \times N_{w^{\prime}}$ search directions, namely $\mathbf{v}^{l, 1}, \mathbf{v}^{u, 1}, \ldots, \mathbf{v}^{l, i}, \quad \mathbf{v}^{u, i}, \ldots, \mathbf{v}^{l, N_{w^{\prime}}}, \mathbf{v}^{u, N_{w^{\prime}}}$ are defined, where $\mathbf{v}^{l, i}, i=1,2, \ldots, N_{w^{\prime}}$ and $\mathbf{v}^{u, i}, i=1,2, \ldots, N_{w^{\prime}}$ represent the search directions setting out from the lower and upper bound points $\mathbf{L}$ and $\mathbf{U}$, respectively. Note that the search direction is always a line setting out from the lower or upper boundary point through the chosen solutions regardless the dimension of the search space, which can be defined as follows:

$$
\begin{gathered}
\mathbf{v}^{l, i}=\mathbf{x}_{j}-\mathbf{L} \\
\mathbf{v}^{u, i}=\mathbf{x}_{j}-\mathbf{U}
\end{gathered}
$$

In Eq. (2), $\mathbf{x}_{j}$ represents the solution that has the minimum distance to the ideal point along the reference vector $\mathbf{w}_{i}^{\prime}$ for the $i$-th vector. For example in Fig. 2(b), $\mathbf{v}^{l, 1}$ and $\mathbf{v}^{u, 1}$ are two promising search directions defined by solution $\mathbf{x}_{1}$ in a threedimensional decision space. The blue surface is the true PS and two red solid triangles are the interaction points between the two direction vectors and the PS in a three-dimensional decision space.

Algorithm 1 presents the pseudocode of the method for defining the search directions. The angles between each individual $i, i=1,2, \ldots,|P|$ of the current parent population and all reference vectors in $\mathbf{W}^{\prime}$ will be calculated, and individual $i$ will be assigned to its closest reference vector that has the minimal angle (lines 2-5). Then for each reference vector $\mathbf{w}^{\prime}{ }_{i}$ that has been assigned at least one individual, the solution having the minimum projected distance to the ideal point in the objective space will be stored in set $S$ (lines 6-11). For a reference vector without any individual being assigned to, its closest individual assigned to another reference vector but is not included in $S$, will be assigned to this reference vector and then is stored in $S$ (lines 12-17). As a result, a total of $N_{w^{\prime}}$ solutions will be chosen and stored in $S$, each of which will be used for defining two search directions.

2) Directed solution sampling : Given a set of search directions $\mathbf{V}=\left\{\mathbf{v}^{l, 1}, \mathbf{v}^{u, 1}, \ldots, \mathbf{v}^{l, i}, \mathbf{v}^{u, i}, \ldots, \mathbf{v}^{l, N_{w^{\prime}}}, \mathbf{v}^{u, N_{w^{\prime}}}\right\}$, we are now going to generate a set of solutions along each of the search directions to identify solutions that can accelerate convergence. Each search direction is a line setting out from the lower or upper boundary point, and consequently there will be three different situations for the positional relationship between the line and the Pareto set, i.e., no intersection, one intersection, or more than one intersection. Fig. 3 gives an example of a two-dimensional problem to show three cases. Theoretically, we can use an optimization algorithm to find the most promising solution along each search direction. For example, in Figs. 3(a) and 3(b), we can utilize an optimization method to find a solution that is closest to the Pareto set on each search direction so that it can be used to guide offspring generation in LMOEA-DS. However, when there is more than one solution on a search direction, such as the case in Fig. 3(c), a search algorithm may fail to find the

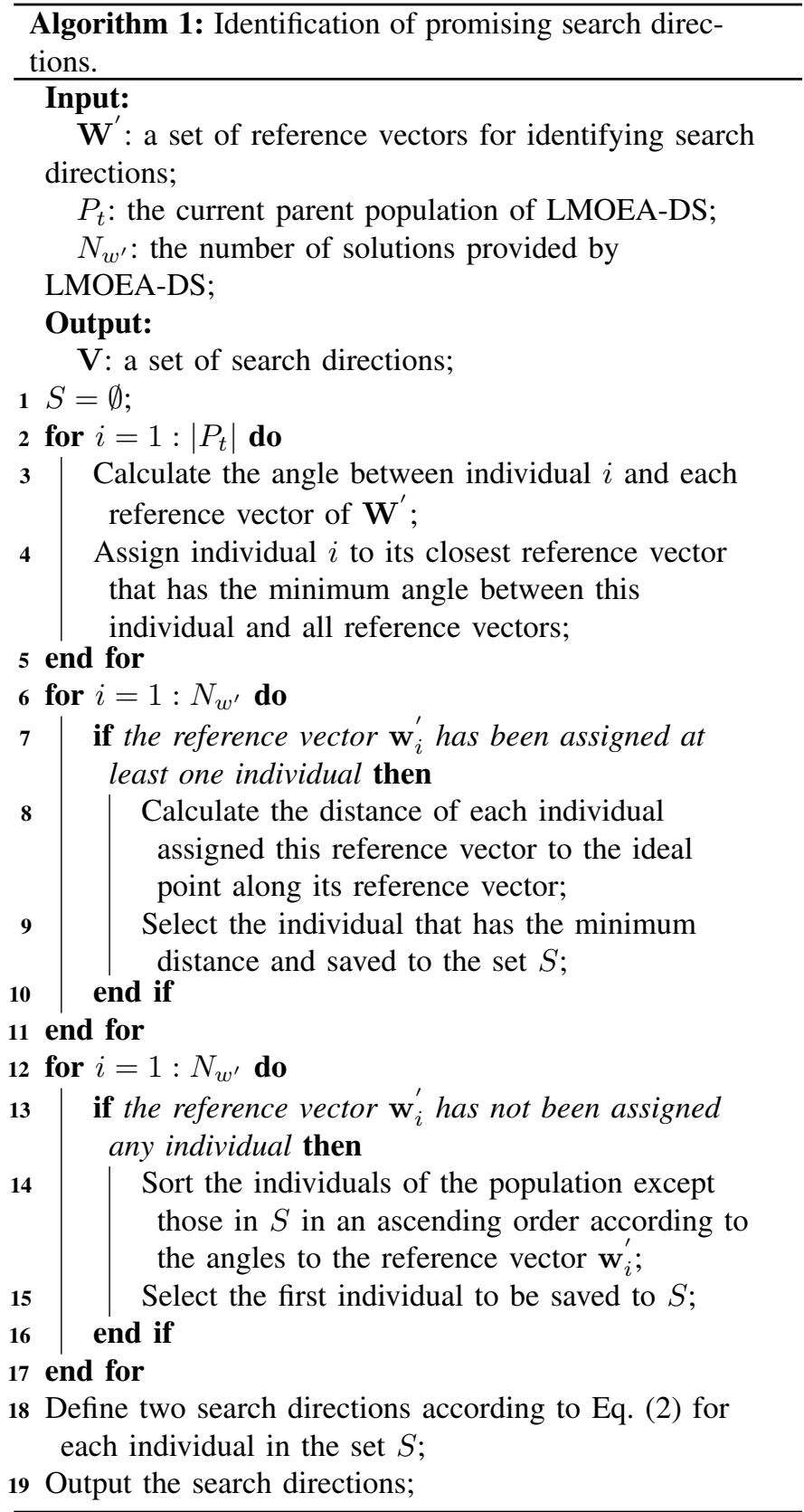

most promising guiding solutions it can easily end up with one solution that is closet to initial search point. Therefore, in this work, we decide to randomly sample multiple solutions along the search directions and then find the non-dominated solutions from all sampled solutions. The benefit of directed sampling over optimization is that directed sampling is able to maintain a higher degree of diversity of the guiding solutions for speeding up the convergence of LMOEA-DS.

Assume solutions $\mathbf{x}_{j}^{l, i}$ and $\mathbf{x}_{k}^{u, i}$ are generated along the search directions $\mathbf{v}^{l, i}$ and $\mathbf{v}^{u, i}$, respectively, which can be formulated as follows:

$$
\begin{aligned}
& \mathbf{x}_{j}^{l, i}=\mathbf{L}+s_{j}^{l, i} \cdot \frac{\mathbf{v}^{l, i}}{\left\|\mathbf{v}^{l, i}\right\|} \\
& \mathbf{x}_{k}^{u, i}=\mathbf{U}+s_{k}^{u, i} \cdot \frac{\mathbf{v}^{u, i}}{\left\|\mathbf{v}^{u, i}\right\|}
\end{aligned}
$$




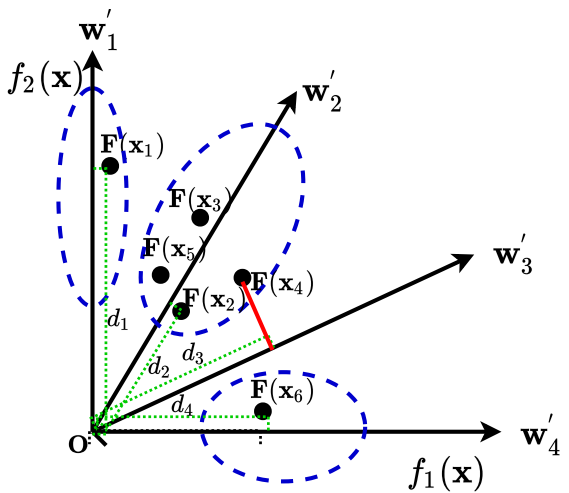

(a) Selection of the solutions for defining search (b) Definition of the search directions. directions

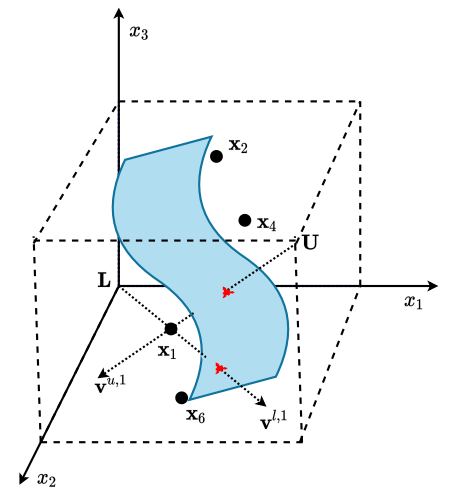

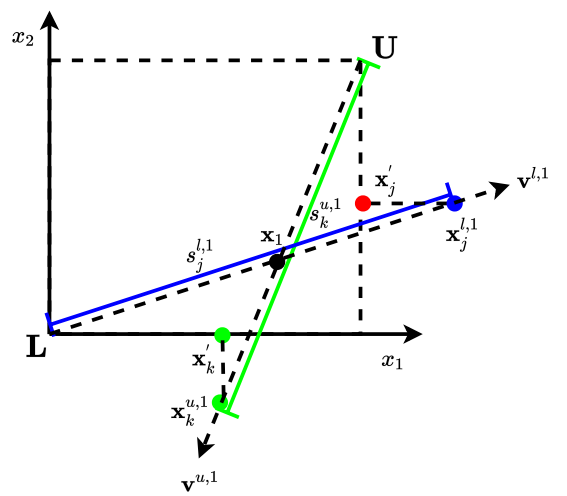

(c) Repair of infeasible solutions.

Fig. 2. (a) Solution selection of a solution for each reference vector that has the minimum projected distance to the ideal point in the objective space, and (b) define two promising search directions based on the selected solution as well as the lower and upper bound solutions in the decision space. (c) An illustration of repairing randomly sampled infeasible solutions, in which any decision variables that exceed the upper or lower bound are projected back to the feasible solutions.

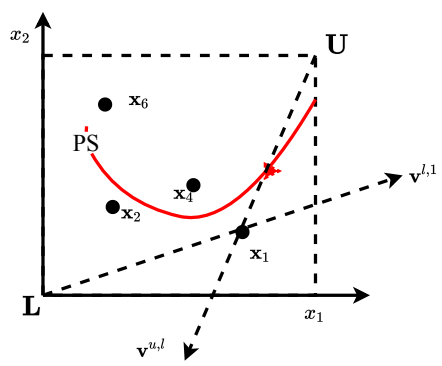

(a) No solution.

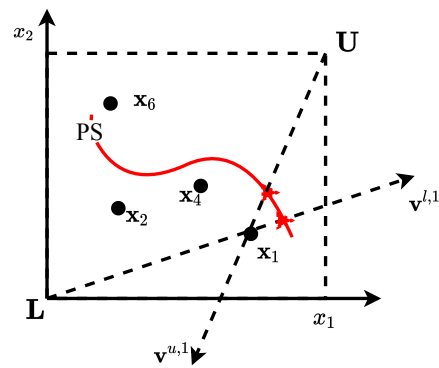

(b) One solution.

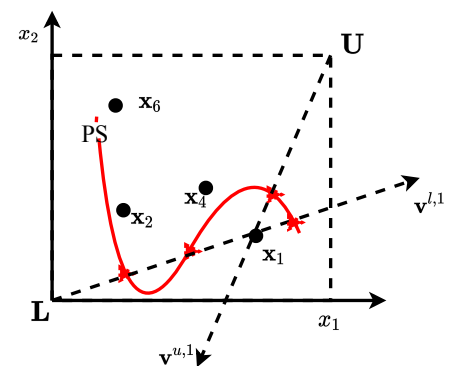

(c) More than one solutions.

Fig. 3. Three cases of the intersection between a search direction $\mathbf{v}^{l, 1}$ and the PS. (a) No solution on the search direction is in the Pareto set; (b) One and only one solution on the search direction is in the Pareto set; and (c) More than one solutions on the search direction are in the Pareto set.

where $s_{j}^{l, i}$ and $s_{k}^{u, i}$ are non-negative coefficients that determine which solutions along the directions $\mathbf{v}^{l, i}$ and $\mathbf{v}^{u, i}$, respectively, will be generated, $j$ and $k$ represent the $j$ - and $k$-th sampling on $\mathbf{v}^{l, i}$ and $\mathbf{v}^{u, i}$, respectively. Note that all solutions generated along these two directions are bounded by $\|\mathbf{U}-\mathbf{L}\|$, where $\|\cdot\|$ is the Euclidean distance. In order to ensure the diversity of the generated solutions, the value of $s_{j}^{l, i}$ and $s_{k}^{u, i}$ are random numbers uniformly distributed in the interval $[0,\|\mathbf{U}-\mathbf{L}\|]$. The influence of the upper bound of the coefficient on the performance of the proposed algorithm is analyzed in Section B of the Supplementary material.

Note however, that some solutions generated by the sampled coefficients will be outside the upper and lower bounds using the $\|\mathbf{U}-\mathbf{L}\|$ defined above. To tackle this problem, all decision variables whose values are outside the lower or upper bound are projected back to the feasible region. Fig. 2(c) gives an illustrative example showing how two solutions generated outside the lower and upper bounds, respectively, are projected back to the feasible region. In Fig. 2(c), $s_{j}^{l, 1}$ and $s_{k}^{u, 1}$ are two coefficients randomly sampled between $[0,\|\mathbf{U}-\mathbf{L}\|]$, resulting two solutions that are outside the feasible region. More specifically, solution $\mathbf{x}_{j}^{l, 1}$ exceeds the upper bound of $x_{1}$ and solution $\mathbf{x}_{k}^{u, 1}$ exceeds the lower bound of $x_{2}$. Therefore, as shown in Fig. 2(c), solutions $\mathbf{x}_{j}^{l, i}$ and $\mathbf{x}_{k}^{u, 1}$ will be projected to points $\mathbf{x}_{j}^{\prime}$ and $\mathbf{x}_{k}^{\prime}$, respectively, in the following way:

$$
\begin{aligned}
x_{j, d}^{\prime} & =\min \left\{x_{j, d}^{l, i}, U_{d}\right\} \\
x_{k, d}^{\prime} & =\max \left\{x_{k, d}^{u, i}, L_{d}\right\}
\end{aligned}
$$

where $i$ indicates the $i$-th reference vector, $j$ and $k$ represent the $j$ - and $k$-th individuals sampled on the search directions $\mathbf{v}^{l, i}$ and $\mathbf{v}^{u, i}$, respectively, and $d=1,2, \ldots, D$ is $d$-th decision variable.

3) Generation of guiding solutions: As described above, a number of solutions will be generated along the two search directions for each reference vector by randomly generating a number of coefficients uniformly distributed between 0 and $\|\mathbf{U}-\mathbf{L}\|$. Assume $N_{s}$ solutions will be sampled for each search direction of each vector, then altogether $2 \times N_{s} \times N_{w^{\prime}}$ solutions will be sampled in total. These solutions are put together and non-dominated sorting is performed to find out the non-dominated solutions. These non-dominated solutions stored in archive $S_{G}$ will be eventually used as the guiding solutions in the reproduction of the LMOEA-DS for speeding up the convergence.

\section{Double Reproduction and Complementary Environmental Selection}

In the proposed method, the guiding solutions are used in the reproduction of LMOEA-DS so as to speed up the conver- 
gence. However, it is often important in an MOEA to maintain a good balance between convergence and diversity. For this reason, a double reproduction process and a complementary environmental selection strategy are introduced in LMOEADS, the details of which are described in the following.

In the first reproduction, each individual in the parent population $P_{t}$ will be selected to do a crossover with a randomly selected guiding solution, thereby generating $\left|P_{t}\right|$ candidate solutions, where $\left|P_{t}\right|$ is the population size. A mutation operator will further be performed on these solutions to get intermediate offspring population $O_{t}^{\prime}$. $P_{t}$ is then combined with $O_{t}^{\prime}$ and the guiding solution set $S_{G}$ to form a combined population $C_{t}^{\prime}=P_{t} \cup O_{t}^{\prime} \cup S_{G}$, on which the first environmental selection is applied, resulting in an intermediate parent population $P_{t}^{\prime}$. The environmental selection applied here is a complementary selection strategy, which is to be detailed later on. Once the first environmental selection is conducted, a second reproduction operation is implemented by performing crossover on the intermediate parent population $P_{t}^{\prime}$ and mutation sequentially to produce the offspring $O_{t}$, and finally the parent population $P_{t+1}$ for the next generation will be obtained by performing the same environmental selection on the combined population $C_{t}=P_{t}^{\prime} \cup O_{t}$.

The pseudocode of the environmental selection adopted in this work is given in Algorithm 2. From Algorithm 2, we can see that the environmental selection strategy complementarily uses the decomposition based method (lines 4-7) and the dominance based method (lines 10-12). The objective values of each individual in the combined population $C_{t}$ will be normalized at first and assigned to their closest reference vector for decomposition based selection. The decomposition based environmental selection will be utilized if the number of reference vectors that have been assigned at least one solution to is not less than a threshold $N_{\epsilon}$. Otherwise the elitist non-dominated sorting proposed in NSGA-II [12] will be employed for environmental selection. The complementary selection strategy ensures that the number of selected offspring will not be too small, and therefore a certain degree of population diversity can be maintained.

The decomposition based selection strategy is performed according to the performance measure described in Eq. (5):

$$
\Psi_{i}=\frac{\cos \theta_{i, j}}{d_{i}}
$$

where $\cos \theta_{i, j}=\frac{\mathbf{F}\left(\mathbf{x}_{\mathbf{i}}\right) \cdot \mathbf{w}_{j}}{\left\|\mathbf{F}\left(\mathbf{x}_{\mathbf{i}}\right)\right\|\|\cdot\| \mathbf{w}_{j} \|}$ represents the cosine value of the angle $\theta_{i, j}$ between individual $i$ and its associated reference vector $\mathbf{w}_{j}$, and $d_{i}$ is the Euclidean distance between individual $i$ and the ideal point of the objective space. The dominance based selection method is the same as the one presented in [12]. Non-dominated sorting is performed on the combined population so that a front number is assigned to each solution. Then a crowding distance is calculated for all solutions having the same front number. All solutions are ranked according to their front number in an ascending order and solutions having the same front number are ranked according to their crowding distance in a descending order. A truncation selection is then performed to select $N$ best solutions. The reader is referred to [12] for more details.

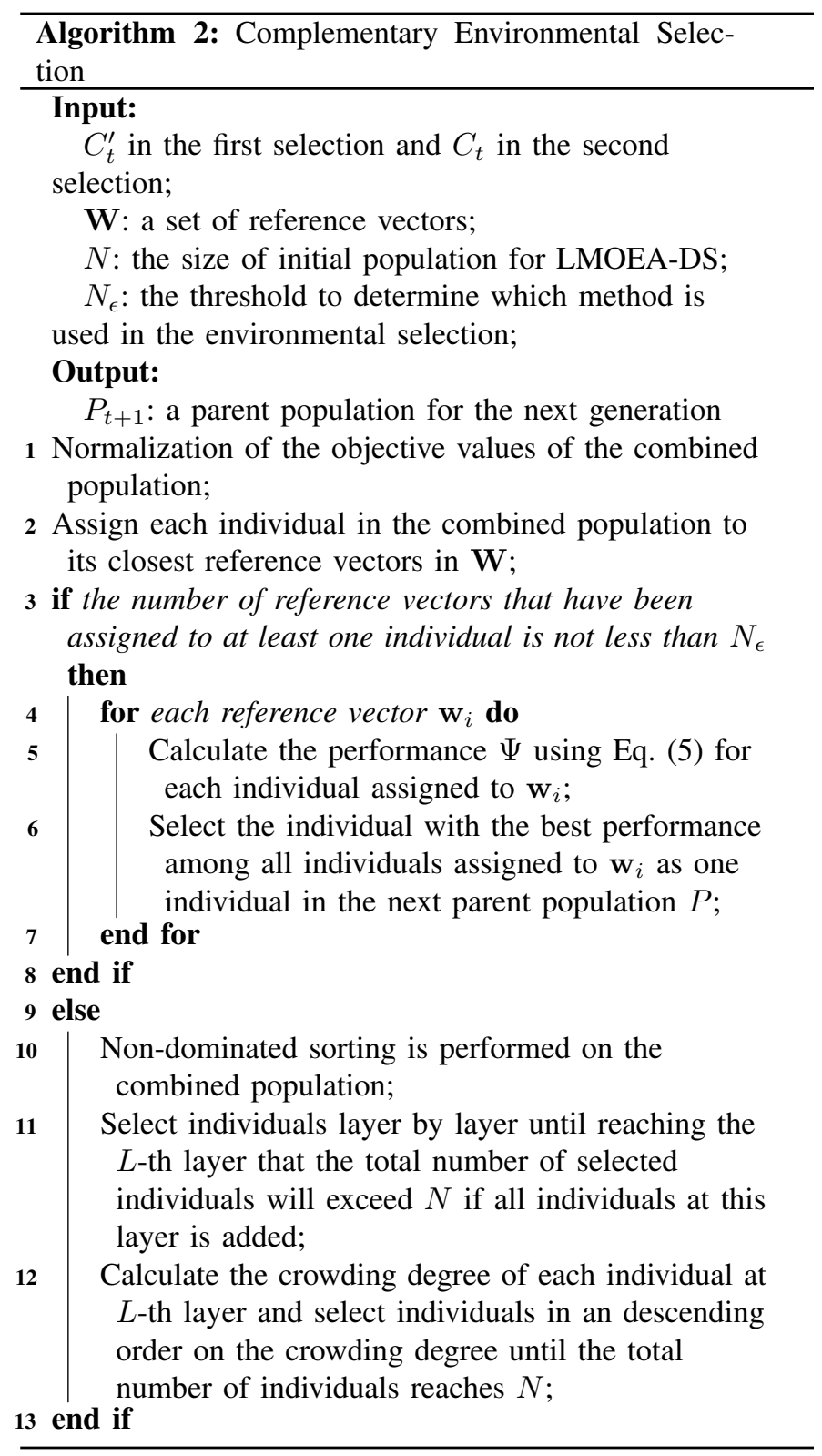

\section{EXPERIMENTAL STUDIES}

To investigate the performance of the proposed method, a set of empirical studies are conducted on nine large-scale multiobjective benchmark problems, namely LSMOP1-9 [75], with 500, 1000, 2000 and 5000 decision variables, respectively. The characteristics of the nine test problems are given in Table I. A commonly used performance indicator, i.e., the inverted generational distance (IGD) [76], is adopted to assess the quality of the solution sets obtained by the algorithms, since IGD is able to account for both accuracy and diversity of a solution set in approximating the true Pareto front [77]. Given a set of solutions $\mathbf{P}$, the IGD value is calculated as follows:

$$
\operatorname{IGD}\left(\mathbf{P}, \mathbf{P}^{*}\right)=\frac{\sum_{\mathbf{x}^{*} \in \mathbf{P}^{*}} d\left(\mathbf{x}^{*}, \mathbf{P}\right)}{\left|\mathbf{P}^{*}\right|}
$$

where $\mathbf{P}^{*}$ is a set of uniformly distributed reference points, $d\left(\mathbf{x}^{*}, \mathbf{P}\right)$ is the minimum Euclidean distance between a reference solution $\mathbf{x}^{*}$ in $\mathbf{P}^{*}$ and all solutions in $\mathbf{P} .\left|\mathbf{P}^{*}\right|$ is the 

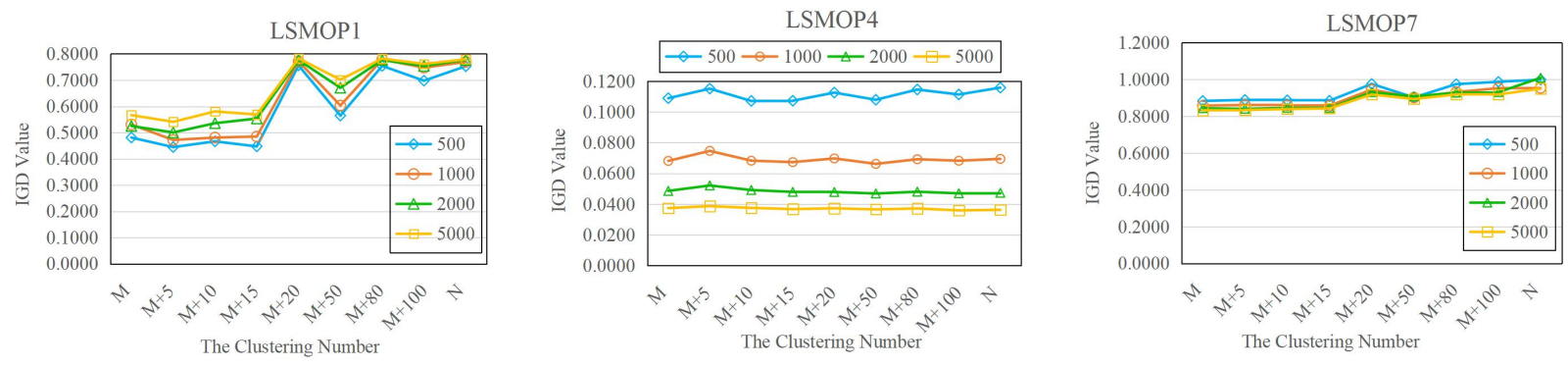

Fig. 4. The parameter analysis of the clustering number $N_{w^{\prime}}$

TABLE I

CHARACTERISTICS OF LSMOP [75] TEST PROBLEMS

\begin{tabular}{c|c|c|c}
\hline \multirow{2}{*}{ Problem } & \multicolumn{3}{|c}{ Characteristics } \\
\cline { 2 - 4 } & Modality & Shape & Separability \\
\hline LSMOP1 & Unimodal & Linear & Fully Separable \\
\hline LSMOP2 & Mixed & Linear & Partially Separable \\
\hline LSMOP3 & Multi-modal & Linear & Mixed \\
\hline LSMOP4 & Mixed & Linear & Mixed \\
\hline LSMOP5 & Unimodal & Convex & Fully Separable \\
\hline LSMOP6 & Mixed & Convex & Partially Separable \\
\hline LSMOP7 & Multi-modal & Convex & Mixed \\
\hline LSMOP8 & Mixed & Convex & Mixed \\
\hline LSMOP9 & Mixed & Disconnected & Fully Separable \\
\hline
\end{tabular}

size of $\mathbf{P}^{*}$. Note that the lower the IGD value is, the better the quality of the obtained optimal solutions.

In the remainder of this section, we will first describe the settings used in the experiments. Then some empirical analysis on the contributions of different components in LMOEA-DS are given. Finally, the method is compared to five state-ofthe-art algorithms on nine bi- and three-dimensional LSMOPs with up to 5000 decision variables.

\section{A. Experimental settings}

Each algorithm under comparison is independently run 20 times on each test instance, and the terminal condition, i.e., the allowed maximum number of objective evaluations (denoted as FEmax), is set to 80000 . To calculate the IGD value of the solution set obtained by each algorithm, 10000 uniformly distributed reference points are sampled on the Pareto front of each test problem [39], which is the same as given in [78]. The Wilcoxon rank-sum test [79] with Bonferroni correction is applied at a significance level of 0.05 to assess whether the performance of a solution set obtained by one of the two compared algorithms is statistically different to another [44], [80]. Symbols ' + ', ' $\approx$ ' and ' - ' indicate that the results obtained by the proposed LMOEA-DS is statistically better than, comparable to, or worse than the compared algorithm, respectively.

In our method, a real-coded genetic algorithm (GA) is adopted to optimize the large-scale multi-objective problem. The initial population size of the GA is set to $N=153$. The simulated binary crossover (SBX) [1] and polynomial mutation (PM) [1], [81] are used as the reproduction operators. For the SBX, the distribution index $\eta_{c}$ and the crossover probability $p_{c}$ are set to 20 and 0.9 , respectively. The distribution index $\eta_{m}$ and mutation probability $p_{m}$ for the PM are set to 20 and $1 / D$, respectively, where $D$ is the number of decision variables. The number of solutions to be randomly generated along each search direction is set to 30 . The threshold $N_{\epsilon}$ is set to $2 N / 3$ to determine which selection strategy is used in the environmental selection.

Note that the number of clusters used to cluster the reference vectors $\mathbf{W}$ plays an important role in the proposed method. The larger the cluster number is, the more search directions will be defined and consequently, a larger number of objective evaluations will be consumed. Therefore, we conduct some pilot studies with different settings for the number of clusters in order to find out a proper setting of this parameter. Fig. 4 gives the mean IGD values of the solution sets obtained by LMOEA-DS on 20 independently runs using different cluster numbers on LSMOP1, LSMOP4 and LSMOP7 with up to 5000 decision variables. From Fig. 4, we can see that LMOEADS can obtain better results when the number of clusters is not more than $M+15$, and there is no clear differences when the cluster number is between $M+5$ and $M+15$. Thus, $M+10$ is adopted in all experiments in the subsequent comparative studies.

The parameter settings used in all compared algorithms are the same to those given in their papers except that for WOFNSGA-II [65], the number of evaluations are set to 800 and 400 , respectively, for the optimization of the original problem and of each transformed problem to meet the maximum number of objective evaluations.

\section{B. Analysis of the effects of the individual components}

In this section, we conduct a set of experiments on nine LSMOP test problems given in Table I with 500, 1000, 2000, 5000 dimensions to show the efficiency and effectiveness of different components designed for LMOEA-DS.

1) The effects of the directed sampling method: In our method, a number of solutions will be randomly generated along two defined search directions for each reference vector. The non-dominated solutions in the set of the randomly solutions will be used as the guiding solutions to accelerate the convergence to the Pareto front. In order to examine the effectiveness of the directed sampling strategy, we compare the directed sampling (DS) method with one of its variants, called DS-inbound, and the method proposed in LSMOF [66], 
called hypervolume optimization (HO), respectively, on threeobjective LSMOP test problems with 500, 1000, 2000 and 5000 decision variables. The main difference between DS and DS-inbound lies in their repairing strategy for handling infeasible solutions generated by the directed sampling. In DS-inbound, we first determine the bound along the given search direction, and then uniformly sample solutions within the bound. Different to random sampling along the search direction, HO formulates a single-objective optimization problem to obtain a set of guiding solutions. The optimization problem aims to optimize the coefficients for all defined search directions to maximize the hypervolume (HV) of the solutions set generated by the optimized coefficients. In order to make a fair comparison, the population is set to 10 and the optimization is conducted for two generations so that the total number of fitness evaluations consumed by HO, DS and DS-inbound is same at each generation.

Tables II and III present the statistical results on nine LSMOP test problems with up to 5000 dimensions obtained by the proposed LMOEA-DS, LMOEA-DS-inbound and LMOEA-HO, respectively. From Table II, we can see that compared to LMOEA-DS-inbound, in which all solutions are limited within the bound along the search direction, the proposed method can obtain better or comparable results on all 36 problems except LSMOP9 of dimension 5000. The results show that the method adopted in LMOEA-DS to repair infeasible solutions is more effective. Compared to LMOEAHO, we can see that LMOEA-DS is worse only on LSMOP7 with 2000 and 5000 decision variables. Based on the results in both Tables II and III, we can conclude that the proposed DS is effective for finding guiding solutions.

2) The effectiveness of double regeneration: In order to evaluate the performance of the double reproduction strategy, we compare it with a variant with the first reproduction operation only, which is denoted as LMOEA-DS-one. Table IV lists the statistical results obtained by LMOEA-DS and LMOEADS-one on LSMOP1, LSMOP4 and LSMOP7 with up to 5000 dimensions, which are unimodal, mixed, and multimodel problems, respectively. From Table IV, we can see that LMOEA-DS wins on $7 / 12$ problems and loses only on $2 / 12$ problems compared to the LMOEA-DS-one, indicating that that the second reproduction is helpful in improving the quality of the obtained solution set. Furthermore, the IGD results obtained by LMOEA-DS-one and LMOEA-DS on all three-objective LSMOP1-9 test problems are given in Table III of the Supplementary material.

3) The effects of complementary environmental selection: The complementary environmental selection strategy adopted in our work aims to ensure that a sufficient number of individuals will be kept in the parent population so that a high degree of diversity of the next parent population will be maintained. In order to verify the effectiveness of the complementary environmental selection, we compare it with two LMOEA-DS variants, one utilizing the decomposition based selection only and the other using the non-dominated sorting based selection only, which are denoted as LMOEA-DS-decomposition, LMOEA-DS-domination, respectively. The statistical results of LMOEA-DS-decomposition, LMOEA-DS-domination and
TABLE II

THE STATISTICAL RESULTS (MEDIAN AND MEDIAN ABSOLUTE DEVIATION) OBTAINED BY LMOEA-DS AND LMOEA-DS-INBOUND ON THREE-OBJECTIVE LSMOP TEST PROBLEMS WITH 500, 1000, 2000 AND

5000 DIMENSIONS. THE BEST MEDIAN RESULT IN EACH ROW IS HIGHLIGHTED.

\begin{tabular}{|c|c|c|c|}
\hline Problem & $\mathrm{D}$ & LMOEA-DS-inbound & LMOEA-DS \\
\hline \multirow{4}{*}{ LSMOP1 } & 500 & $6.4115 \mathrm{e}-01(1.99 \mathrm{e}-02)[+]$ & $4.5498 \mathrm{e}-01(2.41 \mathrm{e}-02)$ \\
\hline & 1000 & $6.8246 \mathrm{e}-01(4.13 \mathrm{e}-02)[+]$ & $4.7643 \mathrm{e}-01(4.11 \mathrm{e}-02)$ \\
\hline & 2000 & $7.3085 \mathrm{e}-01(5.18 \mathrm{e}-02)[+]$ & $4.7684 \mathrm{e}-01(8.15 \mathrm{e}-02)$ \\
\hline & 5000 & $7.4239 \mathrm{e}-01(2.10 \mathrm{e}-02)[+]$ & $5.5356 \mathrm{e}-01(8.04 \mathrm{e}-02)$ \\
\hline \multirow{4}{*}{ LSMOP2 } & 500 & $6.8072 \mathrm{e}-02(8.28 \mathrm{e}-03)[+]$ & $4.4929 \mathrm{e}-02(8.38 \mathrm{e}-04)$ \\
\hline & 1000 & $4.5114 \mathrm{e}-02(7.68 \mathrm{e}-03)[+]$ & $3.8613 \mathrm{e}-02(7.89 \mathrm{e}-04)$ \\
\hline & 2000 & $3.6419 \mathrm{e}-02(1.58 \mathrm{e}-03)[+]$ & $3.4450 \mathrm{e}-02(8.04 \mathrm{e}-04)$ \\
\hline & 5000 & $3.4356 \mathrm{e}-02(6.10 \mathrm{e}-04)[+]$ & $3.3239 \mathrm{e}-02(9.80 \mathrm{e}-04)$ \\
\hline \multirow{4}{*}{ LSMOP3 } & 500 & $8.6056 \mathrm{e}-01(4.53 \mathrm{e}-05)[+]$ & $8.6048 \mathrm{e}-01(3.62 \mathrm{e}-03)$ \\
\hline & 1000 & $8.6059 \mathrm{e}-01(8.34 \mathrm{e}-01)[+]$ & $8.6049 \mathrm{e}-01(1.21 \mathrm{e}-03)$ \\
\hline & 2000 & $8.6062 \mathrm{e}-01(2.51 \mathrm{e}-03)[\approx]$ & $8.6057 \mathrm{e}-01(3.11 \mathrm{e}-04)$ \\
\hline & 5000 & $8.6088 \mathrm{e}-01(1.71 \mathrm{e}-03)[+]$ & $8.6064 e-01(1.45 e-03)$ \\
\hline \multirow{4}{*}{ LSMOP4 } & 500 & $1.4157 \mathrm{e}-01(3.21 \mathrm{e}-03)[+]$ & $1.0818 \mathrm{e}-01(1.78 \mathrm{e}-03)$ \\
\hline & 1000 & $9.3838 \mathrm{e}-02(3.94 \mathrm{e}-03)[+]$ & $6.8001 \mathrm{e}-02(8.82 \mathrm{e}-04)$ \\
\hline & 2000 & $6.5975 \mathrm{e}-02(6.85 \mathrm{e}-03)[+]$ & $4.8670 \mathrm{e}-02(9.49 \mathrm{e}-04)$ \\
\hline & 5000 & $3.8798 \mathrm{e}-02(2.61 \mathrm{e}-03)[+]$ & $3.7368 \mathrm{e}-02(1.04 \mathrm{e}-03)$ \\
\hline \multirow{4}{*}{ LSMOP5 } & 500 & $9.7699 \mathrm{e}-01(1.80 \mathrm{e}+00)[+]$ & $5.3450 \mathrm{e}-01(1.73 \mathrm{e}-02)$ \\
\hline & 1000 & $8.5876 \mathrm{e}-01(1.18 \mathrm{e}+00)[+]$ & $5.3833 \mathrm{e}-01(1.24 \mathrm{e}-02)$ \\
\hline & 2000 & $9.0060 \mathrm{e}-01(1.20 \mathrm{e}+00)[+]$ & $5.3963 \mathrm{e}-01(1.66 \mathrm{e}-03)$ \\
\hline & 5000 & $1.6300 \mathrm{e}+00(7.42 \mathrm{e}-01)[+]$ & $5.3883 \mathrm{e}-01(1.61 \mathrm{e}-02)$ \\
\hline \multirow{4}{*}{ LSMOP6 } & 500 & $1.1803 \mathrm{e}+00(6.02 \mathrm{e}+03)[+]$ & $7.6982 \mathrm{e}-01(2.93 \mathrm{e}-02)$ \\
\hline & 1000 & $1.5130 \mathrm{e}+00(4.13 \mathrm{e}+03)[+]$ & $7.6694 \mathrm{e}-01(2.70 \mathrm{e}-02)$ \\
\hline & 2000 & $1.4335 \mathrm{e}+00(1.65 \mathrm{e}+03)[+]$ & $7.6152 e-01(2.72 e-02)$ \\
\hline & 5000 & $1.6980 \mathrm{e}+00(7.28 \mathrm{e}+02)[+]$ & $7.4373 \mathrm{e}-01(2.71 \mathrm{e}-02)$ \\
\hline \multirow{4}{*}{ LSMOP7 } & 500 & $1.0084 \mathrm{e}+00(1.07 \mathrm{e}+04)[+]$ & $8.8589 \mathrm{e}-01(4.07 \mathrm{e}-03)$ \\
\hline & 1000 & $9.2927 \mathrm{e}-01(6.15 \mathrm{e}+03)[+]$ & $8.5813 \mathrm{e}-01(1.55 \mathrm{e}-03)$ \\
\hline & 2000 & $9.3152 \mathrm{e}-01(1.05 \mathrm{e}+04)[+]$ & $8.4561 \mathrm{e}-01(5.58 \mathrm{e}-04)$ \\
\hline & 5000 & $9.1965 \mathrm{e}-01(1.65 \mathrm{e}+03)[+]$ & $8.3922 \mathrm{e}-01(1.76 \mathrm{e}-03)$ \\
\hline \multirow{4}{*}{ LSMOP8 } & 500 & $9.6354 \mathrm{e}-01(3.98 \mathrm{e}+02)[+]$ & $2.9319 \mathrm{e}-01(4.09 \mathrm{e}-02)$ \\
\hline & 1000 & $1.4595 \mathrm{e}+00(2.28 \mathrm{e}+00)[+]$ & $2.1473 \mathrm{e}-01(2.97 \mathrm{e}-02)$ \\
\hline & 2000 & $1.4905 \mathrm{e}+00(5.28 \mathrm{e}-01)[+]$ & $2.2651 \mathrm{e}-01(1.29 \mathrm{e}-02)$ \\
\hline & 5000 & $1.7374 \mathrm{e}+00(4.62 \mathrm{e}-01)[+]$ & $2.1994 \mathrm{e}-01(1.67 \mathrm{e}-02)$ \\
\hline \multirow{4}{*}{ LSMOP9 } & 500 & $5.8796 \mathrm{e}-01(8.04 \mathrm{e}-03)[\approx]$ & $5.8575 \mathrm{e}-01(1.18 \mathrm{e}-03)$ \\
\hline & 1000 & $5.8680 \mathrm{e}-01(6.59 \mathrm{e}-03)[+]$ & $5.8175 \mathrm{e}-01(1.28 \mathrm{e}-03)$ \\
\hline & 2000 & $5.8292 \mathrm{e}-01(1.24 \mathrm{e}-02)[\approx]$ & $5.8036 \mathrm{e}-01(1.57 \mathrm{e}-03)$ \\
\hline & 5000 & $5.5922 \mathrm{e}-01(1.22 \mathrm{e}-02)[-]$ & $5.7991 \mathrm{e}-01(1.84 \mathrm{e}-03)$ \\
\hline \multicolumn{2}{|l|}{$+/ \approx 1$} & $32 / 3 / 1$ & \\
\hline
\end{tabular}


TABLE III

THE STATISTICAL RESULTS (MEDIAN AND MEDIAN ABSOLUTE DEVIATION) OBTAINED BY LMOEA-DS AND LMOEA-HO ON THREE-OBJECTIVE LSMOP TEST PROBLEMS WITH 500, 1000, 2000 AND 5000 DIMENSIONS. THE BEST MEDIAN RESULT IN EACH ROW IS HIGHLIGHTED.

\begin{tabular}{|c|c|c|c|}
\hline Problem & $\mathrm{D}$ & LMOEA-HO & LMOEA-DS \\
\hline \multirow{4}{*}{ LSMOP1 } & 500 & $4.5505 \mathrm{e}-01(4.45 \mathrm{e}-02)[\approx]$ & $4.5498 \mathrm{e}-01(2.41 \mathrm{e}-02)$ \\
\hline & 1000 & $4.8421 \mathrm{e}-01(4.95 \mathrm{e}-02)[\approx]$ & $4.7643 \mathrm{e}-01(4.11 \mathrm{e}-02)$ \\
\hline & 2000 & $5.2131 \mathrm{e}-01(7.53 \mathrm{e}-02)[\approx]$ & $4.7684 \mathrm{e}-01(8.15 \mathrm{e}-02)$ \\
\hline & 5000 & $5.7129 \mathrm{e}-01(7.95 \mathrm{e}-02)[\approx]$ & $5.5356 \mathrm{e}-01(8.04 \mathrm{e}-02)$ \\
\hline \multirow{4}{*}{ LSMOP2 } & 500 & $4.6390 \mathrm{e}-02(1.04 \mathrm{e}-03)[+]$ & $4.4929 \mathrm{e}-02(8.38 \mathrm{e}-04)$ \\
\hline & 1000 & $3.8432 \mathrm{e}-02(7.05 \mathrm{e}-04)[\approx]$ & $3.8613 \mathrm{e}-02(7.89 \mathrm{e}-04)$ \\
\hline & 2000 & $3.4713 \mathrm{e}-02(4.43 \mathrm{e}-04)[\approx]$ & $3.4450 \mathrm{e}-02(8.04 \mathrm{e}-04)$ \\
\hline & 5000 & $3.3212 \mathrm{e}-02(7.88 \mathrm{e}-04)[\approx]$ & $3.3239 \mathrm{e}-02(9.80 \mathrm{e}-04)$ \\
\hline \multirow{4}{*}{ LSMOP3 } & 500 & $8.6061 \mathrm{e}-01(4.96 \mathrm{e}-04)[+]$ & $8.6048 \mathrm{e}-01(3.62 \mathrm{e}-03)$ \\
\hline & 1000 & $8.6055 \mathrm{e}-01(3.57 \mathrm{e}-03)[\approx]$ & $8.6049 \mathrm{e}-01(1.21 \mathrm{e}-03)$ \\
\hline & 2000 & $8.6056 \mathrm{e}-01(1.20 \mathrm{e}-03)[\approx]$ & $8.6057 \mathrm{e}-01(3.11 \mathrm{e}-04)$ \\
\hline & 5000 & $8.6060 \mathrm{e}-01(1.04 \mathrm{e}-04)[\approx]$ & $8.6064 \mathrm{e}-01(1.45 \mathrm{e}-03)$ \\
\hline \multirow{4}{*}{ LSMOP4 } & 500 & $1.1235 \mathrm{e}-01(1.43 \mathrm{e}-03)[+]$ & $1.0818 \mathrm{e}-01(1.78 \mathrm{e}-03)$ \\
\hline & 1000 & $6.8730 \mathrm{e}-02(1.12 \mathrm{e}-03)[+]$ & $6.8001 \mathrm{e}-02(8.82 \mathrm{e}-04)$ \\
\hline & 2000 & $4.9041 \mathrm{e}-02(7.68 \mathrm{e}-04)[\approx]$ & $4.8670 \mathrm{e}-02(9.49 \mathrm{e}-04)$ \\
\hline & 5000 & $3.7602 \mathrm{e}-02(7.66 \mathrm{e}-04)[\approx]$ & $3.7368 \mathrm{e}-02(1.04 \mathrm{e}-03)$ \\
\hline \multirow{4}{*}{ LSMOP5 } & 500 & $5.3985 \mathrm{e}-01(1.19 \mathrm{e}-02)[\approx]$ & $5.3450 \mathrm{e}-01(1.73 \mathrm{e}-02)$ \\
\hline & 1000 & $5.4045 \mathrm{e}-01(1.03 \mathrm{e}-02)[+]$ & $5.3833 \mathrm{e}-01(1.24 \mathrm{e}-02)$ \\
\hline & 2000 & $5.4059 \mathrm{e}-01(1.02 \mathrm{e}-02)[+]$ & $5.3963 \mathrm{e}-01(1.66 \mathrm{e}-03)$ \\
\hline & 5000 & $5.4057 \mathrm{e}-01(9.99 \mathrm{e}-03)[+]$ & $5.3883 \mathrm{e}-01(1.61 \mathrm{e}-02)$ \\
\hline \multirow{4}{*}{ LSMOP6 } & 500 & $7.7099 \mathrm{e}-01(2.07 \mathrm{e}-02)[\approx]$ & 7.6982e-01(2.93e-02) \\
\hline & 1000 & $7.8703 \mathrm{e}-01(2.74 \mathrm{e}-02)[\approx]$ & $7.6694 \mathrm{e}-01(2.70 \mathrm{e}-02)$ \\
\hline & 2000 & $7.8589 \mathrm{e}-01(2.52 \mathrm{e}-02)[\approx]$ & $7.6152 \mathrm{e}-01(2.72 \mathrm{e}-02)$ \\
\hline & 5000 & $7.9285 \mathrm{e}-01(2.47 \mathrm{e}-02)[+]$ & $7.4373 \mathrm{e}-01(2.71 \mathrm{e}-02)$ \\
\hline \multirow{4}{*}{ LSMOP7 } & 500 & $8.8412 \mathrm{e}-01(3.12 \mathrm{e}-03)[\approx]$ & $8.8589 \mathrm{e}-01(4.07 \mathrm{e}-03)$ \\
\hline & 1000 & $8.5616 \mathrm{e}-01(2.52 \mathrm{e}-03)[-]$ & $8.5813 \mathrm{e}-01(1.55 \mathrm{e}-03)$ \\
\hline & 2000 & $8.4546 \mathrm{e}-01(2.78 \mathrm{e}-03)[\approx]$ & $8.4561 \mathrm{e}-01(5.58 \mathrm{e}-04)$ \\
\hline & 5000 & $8.3901 \mathrm{e}-01(2.83 \mathrm{e}-04)[-]$ & $8.3922 \mathrm{e}-01(1.76 \mathrm{e}-03)$ \\
\hline \multirow{4}{*}{ LSMOP8 } & 500 & $2.9837 \mathrm{e}-01(2.58 \mathrm{e}-02)[\approx]$ & $2.9319 \mathrm{e}-01(4.09 \mathrm{e}-02)$ \\
\hline & 1000 & $3.0210 \mathrm{e}-01(3.54 \mathrm{e}-02)[+]$ & $2.1473 \mathrm{e}-01(2.97 \mathrm{e}-02)$ \\
\hline & 2000 & $2.5855 \mathrm{e}-01(3.55 \mathrm{e}-02)[+]$ & $2.2651 \mathrm{e}-01(1.29 \mathrm{e}-02)$ \\
\hline & 5000 & $2.3600 \mathrm{e}-01(1.31 \mathrm{e}-02)[+]$ & $2.1994 \mathrm{e}-01(1.67 \mathrm{e}-02)$ \\
\hline \multirow{4}{*}{ LSMOP9 } & 500 & $5.8698 \mathrm{e}-01(8.50 \mathrm{e}-04)[+]$ & $5.8575 \mathrm{e}-01(1.18 \mathrm{e}-03)$ \\
\hline & 1000 & $5.8525 \mathrm{e}-01(1.31 \mathrm{e}-03)[+]$ & $5.8175 \mathrm{e}-01(1.28 \mathrm{e}-03)$ \\
\hline & 2000 & $5.8256 \mathrm{e}-01(1.64 \mathrm{e}-03)[+]$ & $5.8036 \mathrm{e}-01(1.57 \mathrm{e}-03)$ \\
\hline & 5000 & $5.8212 \mathrm{e}-01(1.67 \mathrm{e}-03)[+]$ & $5.7991 \mathrm{e}-01(1.84 \mathrm{e}-03)$ \\
\hline+1 & & $15 / 19 / 2$ & \\
\hline
\end{tabular}

LMOEA-DS on LSMOP1, LSMOP4 and LSMOP7 with 500, 1000, 2000 and 5000 dimensions are given in Table V. The IGD results on the three-objective LSMOP1-9 problems with 500, 1000, 2000 and 5000 decision variables are also given in Table IV of the Supplementary material. From Table V, we can see that LMOEA-DS obtains eight competitive and four better results compared to LMOEA-DS-decomposition on 12 problems with up to 5000 dimensions, indicating that LMOEA-DS with decomposition based selection only cannot ensure a sufficient degree of population diversity for many problems. Furthermore, we can see from Table V that the complementary selection strategy in LMOEA-DS can make good use of the decomposition based selection and nondominated sorting based selection, and therefore, achieve a good balance between maintaining population diversity and accelerating convergence.

C. Comparisons with state-of-the-art large-scale multiobjective methods

In order to rigorously evaluate the performance of LMOEADS, we compare LMOEA-DS with several recently proposed large-scale optimization algorithms, namely LSMOF [66], WOF-NSGA-II [65], LMOCSO [67], and MOEA/DVA [62], on LSMOP test problems. Furthermore, an orthogonal crossover algorithm with learning automata (OLEA), proposed in [82], is also used for comparison. In OLEA, the decision variables are divided into groups, aiming to generate fewer promising offspring solutions to reduce the number of objective evaluations. The method was proposed for lowerdimensional multi-objective optimization problems. However, it is also efficient for large-scale multi-objective problems because in OLEA, a set of solutions, which are located close to the Pareto optimal solutions, are chosen for the quantization 
TABLE V

THE STATISTICAL RESULTS (MEDIAN AND MEDIAN ABSOLUTE DEVIATION) OBTAINED BY LMOEA-DS-DECOMPOSITION, LMOEA-DS-DOMINATION AND LMOEA-DS ON THREE-OBJECTIVE LSMOP1, LSMOP4 AND LSMOP7 WITH 500, 1000, 2000 AND 5000 DECISION VARIABLES. THE BEST MEDIAN RESULT IN EACH ROW IS HIGHLIGHTED.

\begin{tabular}{|c|c|c|c|c|}
\hline Problem & $\mathrm{D}$ & LMOEA-DS-decomposition & LMOEA-DS-domination & LMOEA-DS \\
\hline \multirow{4}{*}{ LSMOP1 } & 500 & $4.4066 \mathrm{e}-01(2.59 \mathrm{e}-02)[\approx]$ & $5.5297 \mathrm{e}-01(2.29 \mathrm{e}-02)[+]$ & $4.5498 \mathrm{e}-01(2.41 \mathrm{e}-02)$ \\
\hline & 1000 & $4.6985 \mathrm{e}-01(2.94 \mathrm{e}-02)[\approx]$ & $6.0421 \mathrm{e}-01(2.30 \mathrm{e}-02)[+]$ & $4.7643 \mathrm{e}-01(4.11 \mathrm{e}-02)$ \\
\hline & 2000 & $4.8324 \mathrm{e}-01(3.62 \mathrm{e}-02)[\approx]$ & $6.5004 \mathrm{e}-01(2.28 \mathrm{e}-02)[+]$ & $4.7684 \mathrm{e}-01(8.15 \mathrm{e}-02)$ \\
\hline & 5000 & $4.9964 \mathrm{e}-01(4.11 \mathrm{e}-02)[\approx]$ & $6.6624 \mathrm{e}-01(2.45 \mathrm{e}-02)[+]$ & $5.5356 \mathrm{e}-01(8.04 \mathrm{e}-02)$ \\
\hline \multirow{4}{*}{ LSMOP4 } & 500 & $1.0766 \mathrm{e}-01(1.37 \mathrm{e}-03)[\approx]$ & $1.3539 \mathrm{e}-01(2.28 \mathrm{e}-03)[+]$ & $1.0818 \mathrm{e}-01(1.78 \mathrm{e}-03)$ \\
\hline & 1000 & $6.8479 \mathrm{e}-02(8.16 \mathrm{e}-04)[\approx]$ & $9.2675 \mathrm{e}-02(2.15 \mathrm{e}-03)[+]$ & $6.8001 \mathrm{e}-02(8.82 \mathrm{e}-04)$ \\
\hline & 2000 & $4.8911 \mathrm{e}-02(8.58 \mathrm{e}-04)[\approx]$ & $6.7097 \mathrm{e}-02(1.45 \mathrm{e}-03)[+]$ & $4.8670 \mathrm{e}-02(9.49 \mathrm{e}-04)$ \\
\hline & 5000 & $3.7381 \mathrm{e}-02(6.88 \mathrm{e}-04)[\approx]$ & $5.2832 \mathrm{e}-02(1.85 \mathrm{e}-03)[+]$ & $3.7368 \mathrm{e}-02(1.04 \mathrm{e}-03)$ \\
\hline \multirow{4}{*}{ LSMOP7 } & 500 & $1.3250 \mathrm{e}+00(1.81 \mathrm{e}-02)[+]$ & $8.8454 \mathrm{e}-01(4.68 \mathrm{e}-03)[\approx]$ & $8.8589 \mathrm{e}-01(4.07 \mathrm{e}-03)$ \\
\hline & 1000 & $1.3366 \mathrm{e}+00(2.68 \mathrm{e}-02)[+]$ & $8.5835 \mathrm{e}-01(1.58 \mathrm{e}-03)[\approx]$ & $8.5813 \mathrm{e}-01(1.55 \mathrm{e}-03)$ \\
\hline & 2000 & $1.3406 \mathrm{e}+00(3.01 \mathrm{e}-02)[+]$ & $8.4522 \mathrm{e}-01(7.08 \mathrm{e}-04)[\approx]$ & $8.4561 \mathrm{e}-01(5.58 \mathrm{e}-04)$ \\
\hline & 5000 & $1.3441 \mathrm{e}+00(4.02 \mathrm{e}-02)[+]$ & $8.3902 \mathrm{e}-01(4.72 \mathrm{e}-04)[\approx]$ & $8.3922 \mathrm{e}-01(1.76 \mathrm{e}-03)$ \\
\hline \multicolumn{2}{|c|}{$+/ \approx /-$} & $4 / 8 / 0$ & $8 / 4 / 0$ & - \\
\hline
\end{tabular}

orthogonal crossover with learning automata to guide the search for large-scale optimization.

Tables VI and VII give the statistical results obtained by the compared algorithms on bi- and three-objective LSMOP19 problems with up to 5000 decision variables, respectively. From Tables VI and VII, we can see that compared to MOEA/DVA using the decision variable clustering technique and LMOCSO that uses the new learning strategy, our proposed LMOEA-DS has achieved better results on 36 and 34 , respectively, out of 36 bi-objective problems. Both MOEA/DVA and LMOCSO have obtained better results on one problem only out of all three-objective LSMOP test problems. LMOEA-DS has outperformed WOF-NSGA-II on 25 out of 36 bi-objective LSMOP problems. Furthermore, WOF-NSGA-II was not able to obtain a better result on any of the three-objective LSMOP problems. The LSMOF method utilizes a problem transformation technique to guide the search for large-scale multi-objective optimization. From Tables VI and VII, we can see that our LMOEA-DS has obtained better results on 27 and 29 out of 36 bi-objective and 36 three-objective problems, respectively. Especially, as can be observed from Table VII, LMOEA-DS can achieve better or competitive performance on all three-objective LSMOP benchmark problems when the number of decision variables is larger than 500. Compared to OLEA, which does not fall in any of the four categories of large-scale multi-objective optimization, we can see that LMOEA-DS is underperformed on five bi-objective LSMOP test problems and 11 threeobjective LSMOP test problems, respectively, with up to 5000 dimensions. However, LMOEA-DS has outperfomed OLEA on 26 and 22 out of 36 bi- and three-objective problems. The statistical results of the $\mathrm{HV}$ values obtained by the compared algorithms on bi- and three-objective LSMOP1-9 problems with up to 5000 decision variables are given in Tables VII and VIII, respectively, of the Supplementary material. From these results, we can conclude that the proposed LMOEA-DS can obtain better results than MOEA/DVA, LSMOF, WOFNSGA-II, LMOCSO, and OLEA.

The boxplots of the IGD values obtained by LSMOF, LMOCSO, MOEA/DVA, OLEA, and the proposed LMOEADS on three-objective LSMOP1-9 test problems with 5000 dimensions are presented in Fig. 5. Note that the boxplot of MOEA/DVA is not included in order to display the results of the other algorithms more clearly. The median values of each algorithm have been summarized in Table VII, from which, and from Fig. 5, we can observe that LMOEA-DS can obtain the best median results among the five algorithms under comparison on LSMOP1, LSMOP3, LSMOP5, LSMOP6, LSMOP7, LSMOP8, and LSMOP9 with 5000 decision variables. Furthermore, we can see from Fig. 5 that the interquartile ranges of the IGD values obtained by LMOEADS are relatively small, meaning that it performs robustly on all the nine 5000-dimensional LSMOPs.

Table VIII summarizes the comparative results on all 72 benchmark problems. From Table VIII, we can see that the proposed method has obtained better results on $72,56,57,69$ and 48 out of 72 LSMOP test problems than MOEA/DVA, LSMOF, WOF-NSGA-II, LMOCSO and OLEA, respectively, which shows the promising performance of our proposed method.

\section{CONCLUSION}

To enhance the convergence performance of evolutionary algorithm in solving large-scale multi-objective problems, this paper proposed a method for generating guiding solutions along search directions defined by a few chosen individuals in the current parent population. The guiding solutions are then utilized in a double reproduction process in combination with a complementary selection strategy in an effort to generate well converged and diverse solutions. The experimental results 

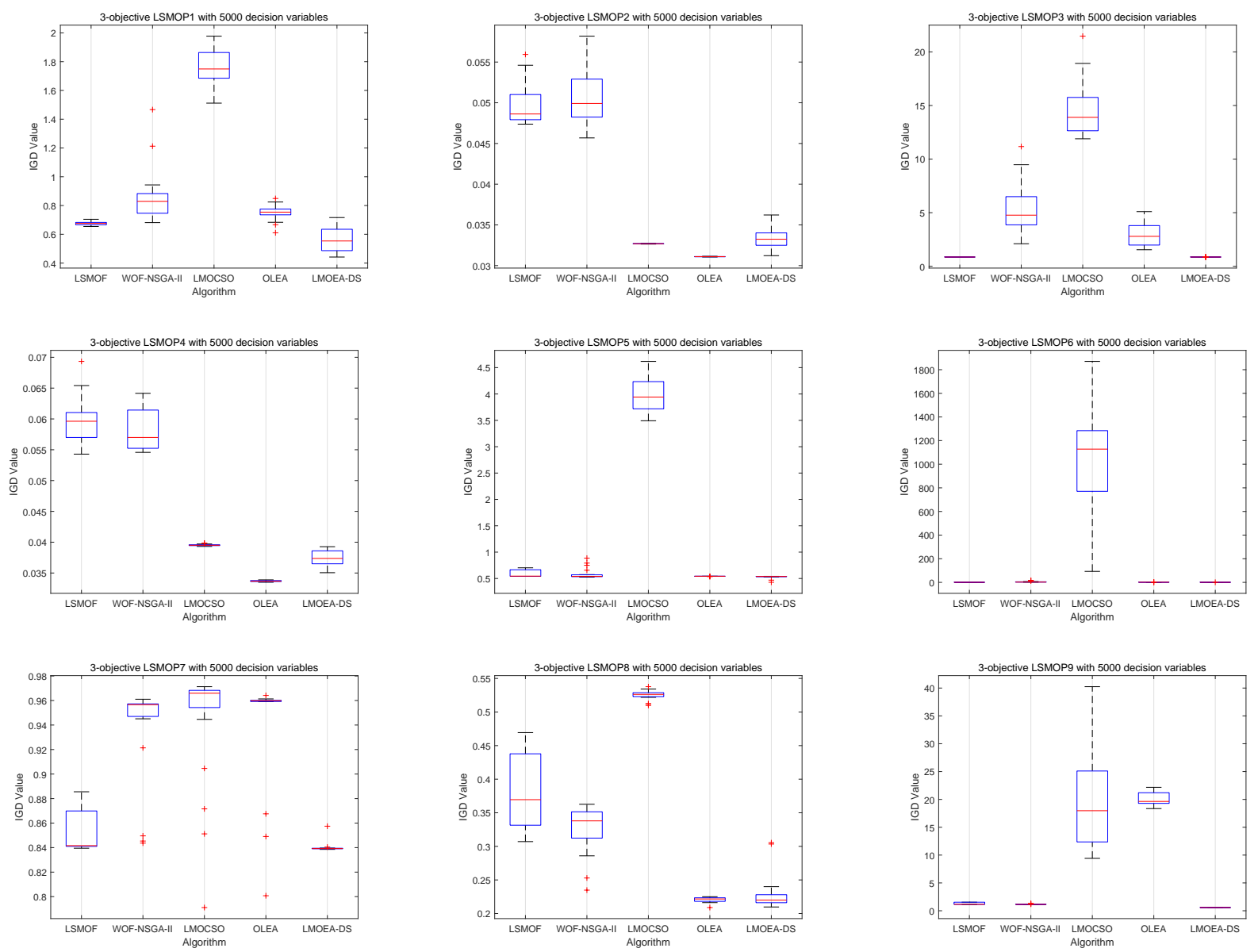

Fig. 5. The boxplots of LMOEA-DS and other four algorithms on three-objective LSMOP1-9 with 5000 decision variables.

confirm that our proposed method is highly competitive in comparson with five state-of-the-art algorithms on bi- and three-objective large-scale multi-objective problems with up to 5000 dimensions. Notably, the proposed algorithm scales particularly well to the increase in the number of decision variables.

The proposed algorithm also suffers from one common weakness of decomposition based algorithms is that their performance heavily depends on the degree of match between the distribution of the reference vectors and the shape of the Pareto fronts of the problem to be solved. In the future, we will make efforts to develop strategies for adapting the reference vectors to achieve more powerful environmental selection during the optimization. In addition, since maintaining a sufficient degree of population diversity is extremely important for large-scale multi-objective optimization problems, we will design new regeneration strategies to enhance the population diversity to further improve the search performance of the proposed algorithm on large-scale multi-objective problems.

\section{ACKNOWLEDGMENT}

This work was supported in part by National Natural Science Foundation of China (Grant No. 61876123), Fund Program for the Scientific Activities of Selected Returned Overseas Professionals in Shanxi Province, Shanxi Province
Science Foundation for Youths (201801D121131), Shanxi Science and Technology Innovation project for Excellent Talents (201805D211028), Postgraduate Education Innovation Project of Shanxi Province (2019SY494) and the China Scholarship Council (CSC).

\section{REFERENCES}

[1] K. Deb, Multi-objective optimization using evolutionary algorithms. New York, NY, USA: John Wiley \& Sons, Inc., 2001.

[2] R. C. Purshouse, "On the evolutionary optimisation of many objectives," IEEE Transactions on Evolutionary Computation, vol. 11, no. 6, pp. 770-784, 2007.

[3] D. Brockhoff, T. Friedrich, N. Hebbinghaus, C. Klein, F. Neumann, and E. Zitzler, "On the effects of adding objectives to plateau functions," IEEE Transactions on Evolutionary Computation, vol. 13, no. 3, pp. 591-603, 2009.

[4] A. Zhou, B. Y. Qu, H. Li, S. Z. Zhao, P. N. Suganthan, and Q. Zhang, "Multiobjective evolutionary algorithms: A survey of the state of the art," Swarm \& Evolutionary Computation, vol. 1, no. 1, pp. 32-49, 2011.

[5] Y. Zhou, Z. Chen, and J. Zhang, "Ranking vectors by means of the dominance degree matrix," IEEE Transactions on Evolutionary Computation, vol. 21, no. 1, pp. 34-51, 2017.

[6] Y. Zhang, D. W. Gong, J. Y. Sun, and B. Y. Qu, "A decompositionbased archiving approach for multi-objective evolutionary optimization," Information Sciences, vol. s 430-431, pp. 397-413, 2018.

[7] J. Maltese, B. M. Ombuki-Berman, and A. P. Engelbrecht, "A scalability study of many-objective optimization algorithms," IEEE Transactions on Evolutionary Computation, vol. 22, no. 1, pp. 79-96, 2018. 
TABLE VI

THE STATISTICAL RESULTS (MEDIAN AND MEDIAN ABSOLUTE DEVIATION) OBTAINED BY LMOEA-DS AND FIVE COMPARED ALGORITHMS ON 500-, 1000-, 2000- AND 5000-D 2-OBJECTIVE LSMOP1-9 PROBLEMS. THE BEST MEDIAN RESULT IN EACH ROW IS HIGHLIGHTED.

\begin{tabular}{|c|c|c|c|c|c|c|c|c|}
\hline Problem & M & $\mathrm{D}$ & MOEA/DVA & LSMOF & WOF-NSGA-II & LMOCSO & OLEA & LMOEA-DS \\
\hline \multirow{4}{*}{ LSMOP1 } & \multirow{4}{*}{2} & 500 & $860 e+00(1.78 \mathrm{e}-01)[+]$ & $6.0526 \mathrm{e}-01(2.81 \mathrm{e}-02)[+]$ & 32e-01(7.91e-02) [+] & $01(1.09 \mathrm{e}-$ & $2.5706 \mathrm{e}-01(2.16 \mathrm{e}-02)[-]$ & $1.03 \mathrm{e}-$ \\
\hline & & 1000 & $0281 \mathrm{e}+01(2.19 \mathrm{e}-01)[+]$ & $6.4223 \mathrm{e}-01(2.07 \mathrm{e}-02)[+]$ & $4.4680 \mathrm{e}-01(8.46 \mathrm{e}-02)[+]$ & $1.5818 \mathrm{e}+00(1.30 \mathrm{e}-01)[+]$ & $3.3678 \mathrm{e}-01(2.46$ & $3.2838 \mathrm{e}-01(7.99 \mathrm{e}-03)$ \\
\hline & & 2000 & $1.1047 \mathrm{e}+01(1.23 \mathrm{e}-01)[+]$ & $6.4302 \mathrm{e}-01(9.72 \mathrm{e}-03)[+]$ & $6.1641 \mathrm{e}-01(6.56 \mathrm{e}-02)[+]$ & $2.0286 \mathrm{e}+00(1.35 \mathrm{e}-01)[+]$ & $4.0885 \mathrm{e}-01(3.89 \mathrm{e}-02)[+]$ & $3.3388 \mathrm{e}-01(6.14 \mathrm{e}-03)$ \\
\hline & & 5000 & $1.1458 \mathrm{e}+01(9.82 \mathrm{e}-02)[+]$ & $6.5744 \mathrm{e}-01(7.74 \mathrm{e}-03)[+]$ & $5.2010 \mathrm{e}-01(9.69 \mathrm{e}-02)[+]$ & $2.3083 e+00(1.36 \mathrm{e}-01)[+]$ & $4.8361 \mathrm{e}-01(5.11 \mathrm{e}-02)[+]$ & $3.3982 \mathrm{e}-01(5.00 \mathrm{e}-03)$ \\
\hline \multirow{4}{*}{ LSMOP2 } & \multirow{4}{*}{2} & 500 & $7.2875 \mathrm{e}-02(1.22 \mathrm{e}-04)[+]$ & $1.9443 \mathrm{e}-02(3.83 \mathrm{e}-04)[+]$ & $1.5334 \mathrm{e}-02(5.18 \mathrm{e}-04)[+]$ & $4.3181 \mathrm{e}-02(6.69 \mathrm{e}-04)[+]$ & $1.6252 \mathrm{e}-02(1.11 \mathrm{e}-03)[+]$ & $1.3724 \mathrm{e}-02(4.01 \mathrm{e}-04)$ \\
\hline & & 1000 & $3.9697 \mathrm{e}-02(1.92 \mathrm{e}-04)[+]$ & $1.3987 \mathrm{e}-02(2.71 \mathrm{e}-04)[+]$ & (.22e- 04$)[+]$ & $3962 \mathrm{e}-02(3.78 \mathrm{e}-04)[+]$ & $8.2990 \mathrm{e}-03(3.74$ & 8.0297 \\
\hline & & 2000 & $2.1937 \mathrm{e}-02(1.51 \mathrm{e}-04)[+]$ & $1.0537 \mathrm{e}-02(2.57 \mathrm{e}-04)[+]$ & $1.1354 \mathrm{e}-02(2.53 \mathrm{e}-04)[+]$ & $1.3406 \mathrm{e}-02(1.66 \mathrm{e}-04)[+]$ & $4.7441 \mathrm{e}-03(1.52 \mathrm{e}-04)[-]$ & $5.0935 \mathrm{e}-03(1.23 \mathrm{e}-04)$ \\
\hline & & 5000 & $1.1257 \mathrm{e}-02(2.64 \mathrm{e}-04)[+]$ & $7.6264 \mathrm{e}-03(2.04 \mathrm{e}-04)[+]$ & 7.3807e-03(3.30e-04) [+] & $6.5956 \mathrm{e}-03(5.19 \mathrm{e}-05)[+]$ & $3.0850 \mathrm{e}-03(6.39 \mathrm{e}-05)[-]$ & $3.5386 \mathrm{e}-03(8.05 \mathrm{e}-05)$ \\
\hline \multirow{4}{*}{ LSMOP3 } & \multirow{4}{*}{2} & 500 & $1.0666 \mathrm{e}+03(6.91 \mathrm{e}+02)[+]$ & $1.5649 \mathrm{e}+00(7.79 \mathrm{e}-04)[-]$ & $1.4971 \mathrm{e}+00(1.23 \mathrm{e}-01)[\approx]$ & $1.2704 \mathrm{e}+00(5.45 \mathrm{e}-01)[-]$ & $1.1303 \mathrm{e}+01(1.8$ & 1.5669 \\
\hline & & 1000 & $8.2434 \mathrm{e}+02(5.53 \mathrm{e}+02)[+]$ & $1.5737 \mathrm{e}+00(3.63 \mathrm{e}-04)[\approx]$ & $1.5725 \mathrm{e}+00(8.52 \mathrm{e}-02)[\approx]$ & $3.8277 \mathrm{e}+00(1.64 \mathrm{e}+00)[+]$ & $1.5351 \mathrm{e}+01(1.64 \mathrm{e}+00)[+]$ & $1.5736 \mathrm{e}+00(7.99 \mathrm{e}-04)$ \\
\hline & & 2000 & $3.3261 \mathrm{e}+02(4.69 \mathrm{e}+02)[+]$ & $1.5772 \mathrm{e}+00(2.31 \mathrm{e}-04)[+]$ & $1.5861 \mathrm{e}+00(1.76 \mathrm{e}+00)[+]$ & $1.0422 \mathrm{e}+01(2.41 \mathrm{e}+00)[+]$ & $1.6861 \mathrm{e}+01(1.03 \mathrm{e}+00)[+]$ & $1.5767 \mathrm{e}+00(8.75 \mathrm{e}-04)$ \\
\hline & & 5000 & $7.2178 \mathrm{e}+02(6.44 \mathrm{e}+02)[+]$ & $1.5792 \mathrm{e}+00(9.04 \mathrm{e}-05)[\approx]$ & $1.3898 \mathrm{e}+00(8.76 \mathrm{e}-02)[-]$ & $1.9829 \mathrm{e}+01(2.68 \mathrm{e}+00)[+]$ & $1.8158 \mathrm{e}+01(1.45 \mathrm{e}+00)[+]$ & $1.5789 \mathrm{e}+00(1.62 \mathrm{e}-03)$ \\
\hline \multirow{4}{*}{ LSMOP4 } & \multirow{4}{*}{2} & 500 & $1.2705 \mathrm{e}-01(3.27 \mathrm{e}-04)[+]$ & $4.8133 \mathrm{e}-02(6.47 \mathrm{e}-04)[+]$ & $4.4211 \mathrm{e}-02(5.39 \mathrm{e}-04)[+]$ & $8.7136 \mathrm{e}-02(8.00 \mathrm{e}-04)[+]$ & $4.1694 \mathrm{e}-02(1.41 \mathrm{e}-03)[\approx]$ & $4.0862 \mathrm{e}-02(5.85 \mathrm{e}-04)$ \\
\hline & & 1000 & $7.5277 \mathrm{e}-02(1.88 \mathrm{e}-04)[+]$ & $2.8534 \mathrm{e}-02(3.87 \mathrm{e}-04)[+]$ & $2.6086 \mathrm{e}-02(6.01 \mathrm{e}-04)[+]$ & $5.1722 \mathrm{e}-02(3.28 \mathrm{e}-04)[+]$ & $2.4541 \mathrm{e}-02(9.09 \mathrm{e}-04)[+]$ & $2.2166 \mathrm{e}-02(3.45 \mathrm{e}-04)$ \\
\hline & & 2000 & $4.3467 \mathrm{e}-02(1.24 \mathrm{e}-04)[+]$ & $1.8825 \mathrm{e}-02(5.24 \mathrm{e}-04)[+]$ & $2.4730 \mathrm{e}-02(2.01 \mathrm{e}-03)[+]$ & $2.9576 \mathrm{e}-02(1.00 \mathrm{e}-04)[+]$ & $1.3421 \mathrm{e}-02(4.43 \mathrm{e}-04)[+]$ & $1.2274 \mathrm{e}-02(1.60 \mathrm{e}-04)$ \\
\hline & & 5000 & $2.1755 \mathrm{e}-02(1.20 \mathrm{e}-04)[+]$ & $1.2809 \mathrm{e}-02(4.27 \mathrm{e}-04)[+]$ & $1.1724 \mathrm{e}-02(3.33 \mathrm{e}-04)[+]$ & $1.4318 \mathrm{e}-02(4.87 \mathrm{e}-05)[+]$ & $6.1598 \mathrm{e}-03(1.68 \mathrm{e}-04)[\approx]$ & $6.1763 \mathrm{e}-03(1.07 \mathrm{e}-04)$ \\
\hline \multirow{4}{*}{ LSMOP5 } & \multirow{4}{*}{2} & 500 & $1.9324 \mathrm{e}+01(3.46 \mathrm{e}-01)[+]$ & $7.4209 \mathrm{e}-01(1.11 \mathrm{e}-16)[+]$ & $4.5847 \mathrm{e}-01(8.91 \mathrm{e}-02)[-]$ & $2.0255 \mathrm{e}+00(2.59 \mathrm{e}-01)[+]$ & $5.0112 \mathrm{e}-01(3.15 \mathrm{e}-02)[-]$ & $7.1467 \mathrm{e}-01(5.26 \mathrm{e}-03)$ \\
\hline & & 1000 & $2.2142 \mathrm{e}+01(2.96 \mathrm{e}-01)[+]$ & $7.4209 \mathrm{e}-01(1.11 \mathrm{e}-16)[+]$ & $6.0693 \mathrm{e}-01(7.53 \mathrm{e}-0$ & $3.7939 \mathrm{e}+00(3.55 \mathrm{e}-01)[+]$ & $7.4259 \mathrm{e}-01(5.79 \mathrm{e}-02)[+]$ & 7.1436e-01(5.06e-03) \\
\hline & & 2000 & $2.3666 \mathrm{e}+01(3.37 \mathrm{e}-01)[+]$ & $7.4209 \mathrm{e}-01(1.11 \mathrm{e}-16)[+]$ & $7.4209 \mathrm{e}-01(3.02 \mathrm{e}-03)[+]$ & $5.0262 \mathrm{e}+00(4.15 \mathrm{e}-01)[+]$ & $8.0350 \mathrm{e}-01(3.73 \mathrm{e}-02)[+]$ & 79e-03) \\
\hline & & 5000 & $2.4395 \mathrm{e}+01(2.06 \mathrm{e}-01)[+]$ & $7.4209 \mathrm{e}-01(1.11 \mathrm{e}-16)[+]$ & $7.3429 \mathrm{e}-01(3.19 \mathrm{e}-02)[+]$ & $5.6360 \mathrm{e}+00(4.24 \mathrm{e}-01)[+]$ & $7.7274 \mathrm{e}-01(2.32 \mathrm{e}-02)[+]$ & $7.1410 \mathrm{e}-01(7.19 \mathrm{e}-03)$ \\
\hline \multirow{4}{*}{ LSMOP6 } & \multirow{4}{*}{2} & 500 & $1.4834 \mathrm{e}+03(1.37 \mathrm{e}+03)[+]$ & $3.2011 \mathrm{e}-01(4.40 \mathrm{e}-04)[+]$ & $6.6740 \mathrm{e}-01(3.52 \mathrm{e}-02)[+]$ & $7.8431 \mathrm{e}-01(6.90 \mathrm{e}-03)[+]$ & $7.7864 \mathrm{e}-01(1.48 \mathrm{e}-03)[+]$ & $3.1896 \mathrm{e}-01(6.67 \mathrm{e}-04)$ \\
\hline & & 1000 & $1.1952 \mathrm{e}+03(1.00 \mathrm{e}+03)[+]$ & {$[\approx]$} & $1(8.15 \mathrm{e}-02)[+]$ & $1(1.44 \mathrm{e}-03)[+]$ & $7.6141 \mathrm{e}-01(5.25$ & 3.12 \\
\hline & & 2000 & $1.1512 \mathrm{e}+03(1.38 \mathrm{e}+03)[+]$ & $3.0879 \mathrm{e}-01(1.21 \mathrm{e}-04)[\approx]$ & $6.7175 \mathrm{e}-01(3.31 \mathrm{e}-02)[+]$ & $7.5558 \mathrm{e}-01(1.13 \mathrm{e}-03)[+]$ & $7.5176 \mathrm{e}-01(5.15 \mathrm{e}-02)[+]$ & $3.0878 \mathrm{e}-01(7.37 \mathrm{e}-05)$ \\
\hline & & 5000 & $1.1498 \mathrm{e}+03(1.88 \mathrm{e}+03)[+]$ & $3.0701 \mathrm{e}-01(2.13 \mathrm{e}-03)[+]$ & $6.7004 \mathrm{e}-01(6.38 \mathrm{e}-02)[+]$ & $7.4745 \mathrm{e}-01(1.22 \mathrm{e}-04)[+]$ & 7.4619e-01(8.97e-02) [+] & $3.0699 \mathrm{e}-01(2.89 \mathrm{e}-05)$ \\
\hline \multirow{4}{*}{ LSMOP7 } & \multirow{4}{*}{2} & 500 & $6.3996 \mathrm{e}+04(3.13 \mathrm{e}+03)[+]$ & $1.5019 \mathrm{e}+00(1.17 \mathrm{e}-03)[-]$ & $1.5039 \mathrm{e}+00(5.62 \mathrm{e}-02)[-]$ & $1.6290 \mathrm{e}+02(7.84 \mathrm{e}+01)[+]$ & $1.5601 \mathrm{e}+00(6.26 \mathrm{e}$ & $1.19 \mathrm{e}-03)$ \\
\hline & & 1000 & $7.8286 \mathrm{e}+04(2.80 \mathrm{e}+03)[+]$ & $1.5101 \mathrm{e}+00(3.23 \mathrm{e}-04)[-]$ & $1.5128 \mathrm{e}+00(9.56 \mathrm{e}-02)[\approx]$ & $2.3653 \mathrm{e}+03(7.26 \mathrm{e}+02)[+]$ & $1.5468 \mathrm{e}+00(3.88 \mathrm{e}-02)[+]$ & $1.5119 \mathrm{e}+00(1.21 \mathrm{e}-03)$ \\
\hline & & 2000 & $8.7518 \mathrm{e}+04(1.39 \mathrm{e}+03)[+]$ & $1.5138 \mathrm{e}+00(3.16 \mathrm{e}-04)[-]$ & $1.5192 \mathrm{e}+00(1.37 \mathrm{e}+00)[+]$ & $4.9912 \mathrm{e}+03(1.11 \mathrm{e}+03)[+]$ & $1.5450 \mathrm{e}+00(1.45 \mathrm{e}-02)[+]$ & $1.5147 \mathrm{e}+00(6.13 \mathrm{e}-04)$ \\
\hline & & 5000 & $9.1438 \mathrm{e}+04(1.35 \mathrm{e}+03)[+]$ & $1.5157 \mathrm{e}+00(3.57 \mathrm{e}-04)[-]$ & $1.5191 \mathrm{e}+00(7.88 \mathrm{e}-02)[\approx]$ & $6.7201 \mathrm{e}+03(1.62 \mathrm{e}+03)[+]$ & $1.5419 \mathrm{e}+00(8.13 \mathrm{e}-01)[+]$ & $1.5164 \mathrm{e}+00(4.99 \mathrm{e}-04)$ \\
\hline \multirow{4}{*}{ LSMOP8 } & \multirow{4}{*}{2} & 500 & $1.6204 \mathrm{e}+01(4.11 \mathrm{e}-01)[+]$ & $7.4209 \mathrm{e}-01(1.11 \mathrm{e}-16)[+]$ & $4.0305 \mathrm{e}-01(1.38 \mathrm{e}-01)[-]$ & $1.5896 \mathrm{e}+00(2.46 \mathrm{e}-01)[+]$ & $4.2698 \mathrm{e}-01(8.40 \mathrm{e}-02)[-]$ & 7.0130e-01(1.67e-02) \\
\hline & & 1000 & $1.8883 \mathrm{e}+01(3.16 \mathrm{e}-01)[+]$ & $7.4209 \mathrm{e}-01(1.11 \mathrm{e}-16)[+]$ & $6.1110 \mathrm{e}-01(1.56 \mathrm{e}-01)[\approx]$ & $3.2331 \mathrm{e}+00(3.25 \mathrm{e}-01)[+]$ & $7.4614 \mathrm{e}-01(1.57 \mathrm{e}-02)[+]$ & $7.0728 \mathrm{e}-01(1.00 \mathrm{e}-02)$ \\
\hline & & 2000 & $2.0118 \mathrm{e}+01(2.11 \mathrm{e}-01)[+]$ & $7.4209 \mathrm{e}-01(1.11 \mathrm{e}-16)[+]$ & $7.4209 \mathrm{e}-01(2.08 \mathrm{e}-02)[+]$ & $4.0539 \mathrm{e}+00(4.17 \mathrm{e}-01)[+]$ & $7.5238 \mathrm{e}-01(1.74 \mathrm{e}-02)[+]$ & $7.0942 \mathrm{e}-01(1.65 \mathrm{e}-02)$ \\
\hline & & 5000 & $2.0839 \mathrm{e}+01(8.24 \mathrm{e}-02)[+]$ & $7.4209 \mathrm{e}-01(1.11 \mathrm{e}-16)[+]$ & $6.7999 \mathrm{e}-01(8.86 \mathrm{e}-02)[\approx]$ & $4.2317 \mathrm{e}+00(2.69 \mathrm{e}-01)[+]$ & $7.4544 \mathrm{e}-01(6.95 \mathrm{e}-03)[+]$ & $7.0954 \mathrm{e}-01(1.02 \mathrm{e}-02)$ \\
\hline \multirow{4}{*}{ LSMOP9 } & \multirow{4}{*}{2} & 500 & $4.3027 \mathrm{e}+01(1.55 \mathrm{e}+00)[+]$ & $8.0878 \mathrm{e}-01(6.89 \mathrm{e}-04)[+]$ & $8.0997 \mathrm{e}-01(1.76 \mathrm{e}-04)[+]$ & $7.7112 \mathrm{e}-01(8.66 \mathrm{e}-02)[\approx]$ & $7.4164 \mathrm{e}-01(1.72 \mathrm{e}-01)[\approx]$ & $7.9250 \mathrm{e}-01(4.19 \mathrm{e}-02)$ \\
\hline & & 1000 & $5.2526 \mathrm{e}+01(1.27 \mathrm{e}+00)[+]$ & $8.0752 \mathrm{e}-01(1.36 \mathrm{e}-03)[+]$ & $8.0913 \mathrm{e}-01(6.82 \mathrm{e}-04)[+]$ & $2.9507 \mathrm{e}+00(7.39 \mathrm{e}-01)[+]$ & $8.4399 \mathrm{e}-01(3.84 \mathrm{e}-01)[+]$ & $6.4566 \mathrm{e}-01(3.11 \mathrm{e}-02)$ \\
\hline & & 2000 & $5.8631 \mathrm{e}+01(9.35 \mathrm{e}-01)[+]$ & $8.0538 \mathrm{e}-01(1.48 \mathrm{e}-03)[+]$ & $8.0694 \mathrm{e}-01(4.18 \mathrm{e}-03)[+]$ & $7.0281 \mathrm{e}+00(1.94 \mathrm{e}+00)[+]$ & $1.2944 \mathrm{e}+00(7.48 \mathrm{e}-01)[+]$ & $6.1974 \mathrm{e}-01(1.99 \mathrm{e}-02)$ \\
\hline & & 5000 & $6.1803 \mathrm{e}+01(5.47 \mathrm{e}-01)[+]$ & $8.0324 \mathrm{e}-01(1.95 \mathrm{e}-03)[+]$ & $8.0512 \mathrm{e}-01(2.25 \mathrm{e}-03)[+]$ & $1.0530 \mathrm{e}+01(1.98 \mathrm{e}+00)[+]$ & $2.6056 \mathrm{e}+00(2.61 \mathrm{e}+00)[+]$ & $6.0717 \mathrm{e}-01(1.93 \mathrm{e}-02)$ \\
\hline & & & $36 / 0 / 0$ & $27 / 4 / 5$ & $25 / 6 / 5$ & $/ 1$ & $26 / 5 / 5$ & \\
\hline
\end{tabular}

[8] D. Gong, Y. Han, and J. Sun, "A novel hybrid multi-objective artificial bee colony algorithm for blocking lot-streaming flow shop scheduling problems," Knowledge Based Systems, vol. 148, no. MAY15, pp. 115$130,2018$.

[9] H. Ishibuchi, N. Tsukamoto, and Y. Nojima, "Behavior of evolutionary many-objective optimization," in Tenth International Conference on Computer Modeling and Simulation. IEEE, 2008, pp. 266-271.

[10] Z. He and G. G. Yen, "Ranking many-objective evolutionary algorithms using performance metrics ensemble," in 2013 IEEE Congress on Evolutionary Computation, 2013, pp. 2480-2487.

[11] D. W. Corne, J. D. Knowles, and M. J. Oates, "The Pareto envelopebased selection algorithm for multiobjective optimization," in Parallel Problem Solving from Nature PPSN VI, M. Schoenauer, K. Deb, G. Rudolph, X. Yao, E. Lutton, J. J. Merelo, and H.-P. Schwefel, Eds. Berlin, Heidelberg: Springer Berlin Heidelberg, 2000, pp. 839-848.

[12] K. Deb, A. Pratap, S. Agarwal, and T. Meyarivan, "A fast and elitist multiobjective genetic algorithm: NSGA-II," IEEE Transactions on Evolutionary Computation, vol. 6, no. 2, pp. 182-197, 2002.

[13] M. Laumanns, L. Thiele, K. Deb, and E. Zitzler, "Combining convergence and diversity in evolutionary multiobjective optimization,"
Evolutionary Computation, vol. 10, no. 3, pp. 263-282, 2002.

[14] L. Raisanen and R. M. Whitaker, "Comparison and evaluation of multiple objective genetic algorithms for the antenna placement problem," Mobile Networks \& Applications, vol. 10, no. 1-2, pp. 79-88, 2005.

[15] D. W. Corne and J. D. Knowles, "Techniques for highly multiobjective optimisation: Some nondominated points are better than others," in Conference on Genetic \& Evolutionary Computation, 2007.

[16] L. S. Batista, F. Campelo, F. G. Guimaraes, and J. A. Ramirez, "A comparison of dominance criteria in many-objective optimization problems," in Evolutionary Computation, 2011.

[17] D. Hadka and P. Reed, "Borg: An auto-adaptive many-objective evolutionary computing framework," Evolutionary Computation, vol. 21, no. 2 , p. 231,2013 .

[18] S. Yang, M. Li, X. Liu, and J. Zheng, "A grid-based evolutionary algorithm for many-objective optimization," IEEE Transactions on Evolutionary Computation, vol. 17, no. 5, pp. 721-736, 2013.

[19] M. Li, S. Yang, and X. Liu, "Bi-goal evolution for many-objective optimization problems," Artificial Intelligence, vol. 228, no. C, pp. 4565, 2015.

[20] X. Zhang, Y. Tian, and Y. Jin, “A knee point-driven evolutionary 
TABLE VII

THE STATISTICAL RESULTS (MEDIAN AND MEDIAN ABSOLUTE DEVIATION) OBTAINED BY LMOEA-DS AND FIVE COMPARED ALGORITHMS ON 500-, 1000-, 2000- AND 5000-D 3-OBJECTIVE LSMOP1-9 PROBLEMS. THE BEST MEDIAN RESULT IN EACH ROW IS HIGHLIGHTED.

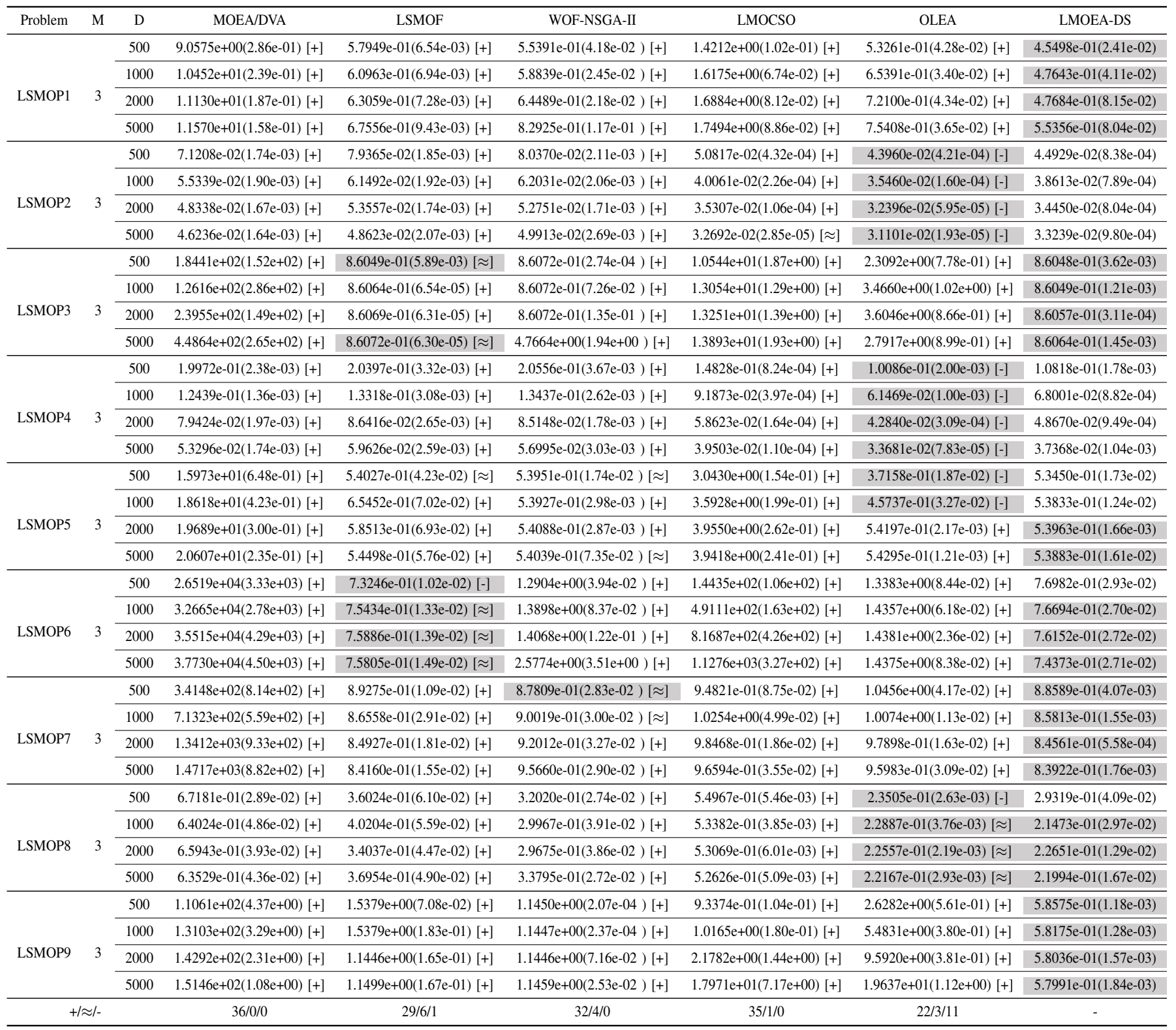

TABLE VIII

A SUMMARY OF THE RESULTS ON LSMOP1-LSMOP9 TEST PROBLEMS, WHERE LMOEA-DS IS BETTER THAN (+), WORST THAN (-) AND COMPARABLE TO $(\approx)$ EACH OF FIVE COMPARED ALGORITHMS ACCORDING TO THE WILCOXON RANK SUM TEST WITH BONFERRONI CORRECTION.

\begin{tabular}{llllll}
\hline $\begin{array}{l}\text { LMOEA- } \\
\text { DS } \\
\text { v.s. }\end{array}$ & MOEA/DVALSMOF & $\begin{array}{l}\text { WOF- } \\
\text { NSGA- } \\
\text { II }\end{array}$ & LMOCSO & OLEA \\
\hline+ & $72 / 72$ & $56 / 72$ & $57 / 72$ & $69 / 72$ & $48 / 72$ \\
\hline$\approx$ & $0 / 72$ & $10 / 72$ & $10 / 72$ & $2 / 72$ & $8 / 72$ \\
\hline- & $0 / 72$ & $6 / 72$ & $5 / 72$ & $1 / 72$ & $16 / 72$ \\
\hline
\end{tabular}

algorithm for many-objective optimization," IEEE Transactions on Evolutionary Computation, vol. 19, no. 6, pp. 761-776, 2015.

[21] Y. Yuan, H. Xu, B. Wang, and X.Yao, "A new dominance relation- based evolutionary algorithm for many-objective optimization," IEEE Transactions on Evolutionary Computation, vol. 20, no. 1, pp. 16-37, 2016.

[22] D. Gong, B. Xu, Y. Zhang, Y. Guo, and S. Yang, "A similaritybased cooperative co-evolutionary algorithm for dynamic interval multiobjective optimization problems," IEEE Transactions on Evolutionary Computation, vol. 24, no. 1, pp. 142-156, Feb 2020.

[23] H. Ishibuchi and T. Murata, "A multi-objective genetic local search algorithm and its application to flowshop scheduling," Computers \& Industrial Engineering, vol. 30, no. 4, pp. 957-968, 1996.

[24] Y. Jin, T. Okabe, and B. Sendho, "Adapting weighted aggregation for multiobjective evolution strategies," in International Conference on Evolutionary Multi-Criterion Optimization. Springer, 2001, pp. 96-110.

[25] T. Murata, H. Ishibuchi, and M. Gen, "Specification of genetic search directions in cellular multi-objective genetic algorithms," in International Conference on Evolutionary Multi-criterion Optimization, 2001.

[26] Q. Zhang and L. Hui, "MOEA/D: A multiobjective evolutionary algorithm based on decomposition," IEEE Transactions on Evolutionary Computation, vol. 11, no. 6, pp. 712-731, 2007.

[27] H. L. Liu, F. Gu, and Q. Zhang, "Decomposition of a multiobjective 
optimization problem into a number of simple multiobjective subproblems," IEEE Transactions on Evolutionary Computation, vol. 18, no. 3, pp. $450-455,2014$

[28] R. Cheng, Y. Jin, M. Olhofer, and B. Sendhoff, "A reference vector guided evolutionary algorithm for many-objective optimization," IEEE Transactions on Evolutionary Computation, vol. 20, no. 5, pp. 773-791, 2016.

[29] N. Beumea and M. Emmerich, "SMS-EMOA: Multiobjective selection based on dominated hypervolume," European Journal of Operational Research, vol. 181, no. 3, pp. 1653-1669, 2007.

[30] E. Zitzler and S. Künzli, "Indicator-based selection in multiobjective search," Lecture Notes in Computer Science, vol. 3242, pp. 832-842, 2004.

[31] K. Bringmann and T. Friedrich, "An efficient algorithm for computing hypervolume contributions," Evolutionary Computation, vol. 18, no. 3, pp. 383-402, 2010

[32] J. Bader and E. Zitzler, "HypE: An algorithm for fast hypervolume-based many-objective optimization," Evolutionary Computation, vol. 19, no. 1, pp. 45-76, 2011.

[33] L. While, L. Bradstreet, and L. Barone, "A fast way of calculating exact hypervolumes," IEEE Transactions on Evolutionary Computation, vol. 16, no. 1, pp. 86-95, 2012.

[34] L. M. S. Russo and A. P. Francisco, "Quick hypervolume," IEEE Transactions on Evolutionary Computation, vol. 18, no. 4, pp. 481-502, 2012.

[35] D. Brockhoff, T. Wagner, and H. Trautmann, "On the properties of the R2 indicator," in Conference on Genetic \& Evolutionary Computation, 2012.

[36] R. H. Gómez and C. A. C. Coello, "MOMBI: A new metaheuristic for many-objective optimization based on the R2 indicator," in Evolutionary Computation, 2013.

[37] H. Trautmann, T. Wagner, and D. Brockhoff, "R2-EMOA: Focused multiobjective search using R2-indicator-based selection," Lecture Notes in Computer Science, vol. 7997, pp. 70-74, 2013.

[38] Y. Tian, R. Cheng, X. Zhang, F. Cheng, and Y. Jin, "An Indicator based multi-objective evolutionary algorithm with reference point adaptation for better versatility," IEEE Transactions on Evolutionary Computation, vol. PP, no. 99, pp. 1-1, 2017.

[39] K. Deb and H. Jain, "An evolutionary many-objective optimization algorithm using reference-point-based nondominated sorting approach, Part I: Solving problems with box constraints," IEEE Transactions on Evolutionary Computation, vol. 18, no. 4, pp. 577-601, 2014.

[40] K. Li, K. Deb, Q. Zhang, and S. Kwong, "An evolutionary manyobjective optimization algorithm based on dominance and decomposition," IEEE Transactions on Evolutionary Computation, vol. 19, no. 5, pp. 694-716, 2015.

[41] D. Gong, J. Sun, and X. Ji, "Evolutionary algorithms with preference polyhedron for interval multi-objective optimization problems," Information Sciences, vol. 233, pp. 141-161, 2013.

[42] M. Rong, D. Gong, Y. Zhang, Y. Jin, and W. Pedrycz, "Multidirectional prediction approach for dynamic multiobjective optimization problems," IEEE Transactions on Cybernetics, vol. 49, no. 9, pp. 3362-3374, 2019

[43] B. Li, J. Li, K. Tang, and X. Yao, "Many-objective evolutionary algorithms: A survey," ACM Computing Surveys, vol. 48, no. 1, pp. $1-35,2015$.

[44] Z. He and G. G. Yen, "Many-objective evolutionary algorithms based on coordinated selection strategy," IEEE Transactions on Evolutionary Computation, vol. 21, no. 2, pp. 220-233, 2017.

[45] X. Cai, Z. Yang, Z. Fan, and Q. Zhang, "Decomposition-based-sorting and angle-based-selection for evolutionary multiobjective and manyobjective optimization," IEEE Transactions on Cybernetics, vol. 47, no. 9, pp. 2824-2837, 2017.

[46] C. Liu, Q. Zhao, B. Yan, S. Elsayed, T. Ray, and R. Sarker, "Adaptive sorting-based evolutionary algorithm for many-objective optimization," IEEE Transactions on Evolutionary Computation, vol. 23, no. 2, pp. 247-257, 2018.

[47] S. Qin, C. Sun, G. Zhang, X. He, and Y. Tan, "A modified particle swarm optimization based on decomposition with different ideal points for many-objective optimization problems," Complex \& Intelligent Systems, vol. 6 , no. 2, pp. 263-274, 2020.

[48] W. W. Hager, D. W. Hearn, and P. M. Pardalos, Large scale optimization: State of the art. Springer Science \& Business Media, 2013.

[49] R. M. Everson and J. E. Fieldsend, "Multiobjective optimization of safety related systems: An application to short-term conflict Alert," IEEE Transactions on Evolutionary Computation, vol. 10, no. 2, pp. 187-198, 2006.
[50] Y. Tian, C. Lu, X. Zhang, K. C. Tan, and Y. Jin, "Solving large-scale multiobjective optimization problems with sparse optimal solutions via unsupervised neural networks," IEEE Transactions on Cybernetics, pp. $1-14,2020$.

[51] Y. Tian, X. Zhang, C. Wang, and Y. Jin, "An evolutionary algorithm for large-scale sparse multiobjective optimization problems," IEEE Transactions on Evolutionary Computation, vol. 24, no. 2, pp. 380-393, 2020.

[52] X. Zhang, Y. Tian, R. Cheng, and Y. Jin, "A decision variable clusteringbased evolutionary algorithm for large-scale many-objective optimization," IEEE Transactions on Evolutionary Computation, vol. 22, no. 1, pp. 97-112, 2018.

[53] L. Parsons, E. Haque, and H. Liu, "Subspace clustering for high dimensional data: A review," Acm Sigkdd Explorations Newsletter, vol. 6, no. 1, pp. 90-105, 2004

[54] R. Cheng and Y. Jin, "A competitive swarm optimizer for large scale optimization," IEEE Transactions on Cybernetics, vol. 45, no. 2, 2015.

[55] —, "A social learning particle swarm optimization algorithm for scalable optimization," Information Sciences, vol. 291, pp. 43-60, 2015.

[56] Q. Yang, W. Chen, T. Gu, H. Zhang, J. D. Deng, Y. Li, and J. Zhang, "Segment-based predominant learning swarm optimizer for large-scale optimization," IEEE Transactions on Cybernetics, vol. 47, no. 9, pp. 2896-2910, Sep. 2017.

[57] M. N. Omidvar, X. Li, Z. Yang, and X. Yao, "Cooperative co-evolution for large scale optimization through more frequent random grouping," in IEEE Congress on Evolutionary Computation, 2010, pp. 1-8.

[58] H. Wang, M. Liang, C. Sun, G. Zhang, and L. Xie, "Multiple-strategy learning particle swarm optimization for large-scale optimization problems," Complex \& Intelligent Systems, 2020.

[59] L. M. Antonio and C. A. C. Coello, "Use of cooperative coevolution for solving large scale multiobjective optimization problems," in IEEE Congress on Evolutionary Computation, 2013.

[60] A. Song, Q. Yang, W. Chen, and J. Zhang, "A random-based dynamic grouping strategy for large scale multi-objective optimization," in 2016 IEEE Congress on Evolutionary Computation (CEC), 2016, pp. 468475.

[61] L. M. Antonio and C. A. C.Coello, "Decomposition-based approach for solving large scale multi-objective problems," in International Conference on Parallel Problem Solving from Nature. Springer, 2016, pp. 525-534.

[62] X. Ma, L. Fang, Y. Qi, X. Wang, L. Li, L. Jiao, M. Yin, and M. Gong, “A multiobjective evolutionary algorithm based on decision variable analyses for multiobjective optimization problems with large-scale variables," IEEE Transactions on Evolutionary Computation, vol. 20, no. 2, pp. 275-298, 2016

[63] H. Chen, X. Zhu, W. Pedrycz, S. Yin, G. Wu, and H. Yan, "PEA: Parallel evolutionary algorithm by separating convergence and diversity for large-scale multi-objective optimization," in 2018 IEEE 38th International Conference on Distributed Computing Systems (ICDCS), 2018. pp. 223-232.

[64] M. Li and J. Wei, "A cooperative co-evolutionary algorithm for largescale multi-objective optimization problems," in Genetic \& Evolutionary Computation Conference Companion, 2018.

[65] H. Zille, H. Ishibuchi, S. Mostaghim, and Y. Nojima, "A framework for large-scale multiobjective optimization based on problem transformation," IEEE Transactions on Evolutionary Computation, vol. 22, no. 2, pp. 260-275, 2018.

[66] C. He, L. Li, Y. Tian, X. Zhang, R. Cheng, Y. Jin, and X. Yao, "Accelerating large-scale multiobjective optimization via problem reformulation," IEEE Transactions on Evolutionary Computation, vol. 23, no. 6, pp. 949-961, 2019.

[67] Y. Tian, X. Zheng, X. Zhang, and Y. Jin, "Efficient large-scale multiobjective optimization based on a competitive swarm optimizer," IEEE Transactions on Cybernetics, pp. 1-13, 2019.

[68] C. He, R. Cheng, C. Zhang, Y. Tian, Q. Chen, and X. Yao, "Evolutionary large-scale multiobjective optimization for ratio error estimation of voltage transformers," IEEE Transactions on Evolutionary Computation, vol. 24 , no. 5, pp. 868-881, 2020.

[69] R. Liu, R. Ren, J. Liu, and J. Liu, "A clustering and dimensionality reduction based evolutionary algorithm for large-scale multi-objective problems," Applied Soft Computing, vol. 89, p. 106120, 2020.

[70] H. Chen, R. Cheng, J. Wen, H. Li, and J. Weng, "Solving large-scale many-objective optimization problems by covariance matrix adaptation evolution strategy with scalable small subpopulations," Information Sciences, vol. 509, pp. 457-469, 2020.

[71] C. He, R. Cheng, and D. Yazdani, "Adaptive offspring generation for evolutionary large-scale multiobjective optimization," IEEE Transactions on Systems, Man, and Cybernetics: Systems, 2020. 
[72] R. Liu, J. Liu, Y. Li, and J. Liu, "A random dynamic grouping based weight optimization framework for large-scale multi-objective optimization problems," Swarm and Evolutionary Computation, vol. 55, p. 100684, 2020.

[73] Y. Zhang, G.-G. Wang, K. Li, W.-C. Yeh, M. Jian, and J. Dong, "Enhancing MOEA/D with information feedback models for large-scale many-objective optimization," Information Sciences, vol. 522, pp. 1-16, 2020.

[74] K. Krishna and M. N. Murty, "Genetic K-means algorithm," IEEE Transactions on Systems, Man, and Cybernetics, Part B (Cybernetics), vol. 29, no. 3, pp. 433-439, 1999.

[75] R. Cheng, Y. Jin, M. Olhofer et al., "Test problems for large-scale multiobjective and many-objective optimization," IEEE Transactions on Cybernetics, vol. 47, no. 12, pp. 4108-4121, 2016.

[76] A. Zhou, Y. Jin, Q. Zhang, and B. Sendhoff, "Combining model-based and genetics-based offspring generation for multi-objective optimization using a convergence criterion," in IEEE Congress on Evolutionary Computation, 2006

[77] P. Czyzżak and A. Jaszkiewicz, "Pareto simulated annealing — a metaheuristic technique for multiple-objective combinatorial optimization," Journal of Multi-Criteria Decision Analysis, vol. 7, no. 1, pp. 34-47, 1998.

[78] Y. Tian, R. Cheng, X. Zhang, and Y. Jin, "PlatEMO: A MATLAB platform for evolutionary multi-objective optimization [Educational Forum]," IEEE Computational Intelligence Magazine, vol. 12, no. 4, pp. 73-87, 2017.

[79] R. G. D. Steel and J. H. Torrie, Principles and procedures of statistics: A biometrical approach. McGraw-Hill Kogakusha, Ltd., 1980, no. 2.

[80] J. Dufner, U. Jensen, and E. Schumacher, Statistik MIT SAS. SpringerVerlag, 2013.

[81] K. Deb and M. Goyal, "A combined genetic adaptive search (GeneAS) for engineering design," Computer Science and Informatics, vol. 26, pp. 30-45, 1996.

[82] C. Dai, Y. Wang, M. Ye, X. Xue, and H. Liu, "An orthogonal evolutionary algorithm with learning automata for multiobjective optimization," IEEE Transactions on Cybernetics, vol. 46, no. 12, pp. 3306-3319, 2016.

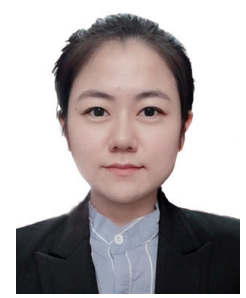

Shufen Qin received the B.Sc. degree in Computer Science and Technology from Jinzhong University, Jinzhong, China, in 2017. She is currently pursuing the $\mathrm{Ph} . \mathrm{D}$. degree with the Department of Electronic Information Engineering, Taiyuan University of Science and Technology, Taiyuan, China.

Her current research interests include particle swarm optimization, multi-/many-objective optimization, large-scale optimization, surrogateassisted evolutionary optimization, and their applications to practical engineering problems.

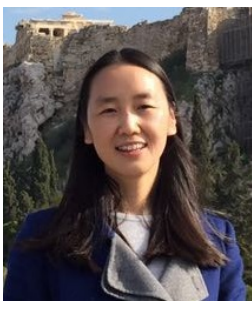

Chaoli Sun (M'15) received the B.Sc. and M.Sc. degrees from Hohai University, Nanjing, China, in 2000 and 2003, respectively, and the Ph.D. degree from Taiyuan University of Science and Technology, Taiyuan, China, in 2011.

She is a Professor of Department of Computer Science and Technology, Taiyuan University of Science and Technology. Her research interests include swarm intelligence, particle swarm optimization, surrogate-assisted evolutionary optimization, and their applications to practical engineering prob-

lems.

Prof. Sun is an Associate Editor of Soft Computing and Complex Intelligent Systems, and was the chair of the Task Force on Data-Driven Evolutionary Optimization of Expensive Problems within the Evolutionary Computation Technical Committee of IEEE Computational Intelligence Society (20152020). She has also served as a reviewer for journals including IEEE TRANSACTIONS ON EVOLUTIONARY COMPUTATION, IEEE TRANSACTIONS ON CYBERNETICS, IEEE TRANSACTIONS ON NEURAL NETWORKS AND LEARNING SYSTEMS, and IEEE Computational Intelligence Magazine.

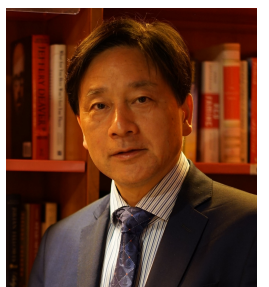

Yaochu Jin (M'98-SM'02-F'16) received the B.Sc., M.Sc., and Ph.D. degrees from Zhejiang University, Hangzhou, China, in 1988, 1991, and 1996, respectively, and the Dr.-Ing. degree from Ruhr University Bochum, Germany, in 2001. He is a Professor in Computational Intelligence, Department of Computer Science, University of Surrey, Guildford, U.K. where he heads the Nature Inspired Computing and Engineering Group.

$\mathrm{He}$ is also a Finland Distinguished Professor funded by the Finnish Funding Agency for Innovation (Tekes) and a Changjiang Distinguished Visiting Professor appointed by the Ministry of Education, China. His research interests lie primarily in the crossdisciplinary areas of computational intelligence, computational neuroscience, and computational systems biology. He is also particularly interested in the application of nature-inspired algorithms to solving real-world optimization, learning and self-organization problems. He has (co)authored over 300 peerreviewed journal and conference papers and been granted eight patents on evolutionary optimization.

Dr Jin is the Editor-in-Chief of the IEEE TRANSACTIONS ON COGNITIVE AND DEVELOPMENTAL SYSTEMS and Complex Intelligent Systems. He is an IEEE Distinguished Lecturer (2017-2019) and was the Vice President for Technical Activities of the IEEE Computational Intelligence Society (2014-2015). He is a recipient of the 2014 and 2016 IEEE Computational Intelligence Magazine Outstanding Paper Award, and the 2017 IEEE Transactions on Evolutionary Computation Outstanding Paper Award. $\mathrm{He}$ is a Fellow of IEEE.

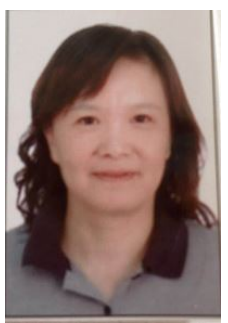

Yin Tan received the B.Sc. degree in Industrial Automation from Taiyuan University of Science and Technology in 1986, and the M.Sc. degree in Control Theory and Application from Tsinghua University in 1994. She is a Professor with the Department of Computer Science and Technology, Taiyuan University of Science and Technology. Her current research interests include intelligent computing, cloud computing and distributed network security.

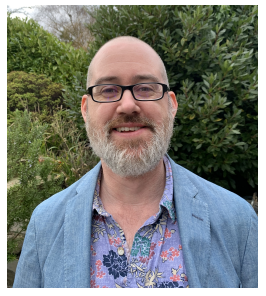

Jonathan Fieldsend (S'00-M'02) received the B.A. degree (Hons.) in Economics from Durham University in 1998, the M.Sc. degree in Computational Intelligence from Plymouth University in 1999, and the Ph.D. degree in Computer Science from the University of Exeter in 2003. He is currently a Professor in Computational Intelligence with the University of Exeter. His research interests include multi-objective optimization, optimization with uncertainty, robust optimization, multi-modal optimization, expensive optimization, machine learning, and data visualization. He is a member of the IEEE Computational Intelligence Society (CIS) and is a Vice Chair of the IEEE CIS Task Force on Multi-modal Optimization and a Vice-Chair of the IEEE CIS Task Force on Data-Driven Evolutionary Optimization of Expensive Problems. He is an Associate Editor of IEEE Transactions on Evolutionary Computation. 